OFR: $72-37$

\title{
An Evaluation of Water-Quality Records for Texas Streams
}

$\frac{5}{5}$
$\frac{5}{7}$
$\frac{5}{7}$

\section{UNITED STATES DEPARTMENT OF THE INTERIOR} GEOLOGICAL SURVEY - WATER RESOURCES DIVISION
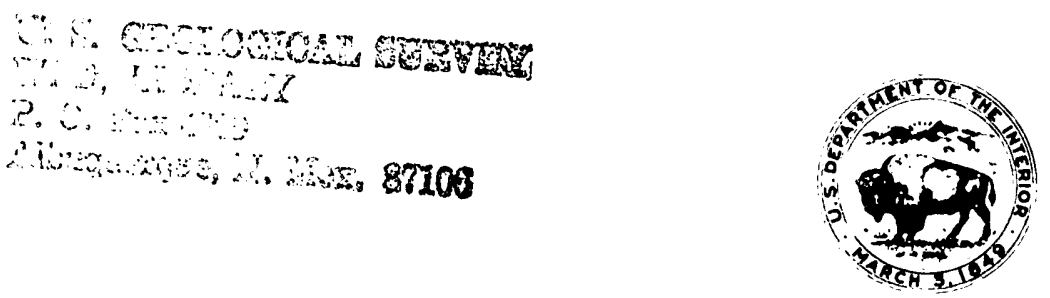


\section{An Evaluation of Water-Quality Records for Texas Streams}

By J. F. Blakey, R. O. Hawkinson, and T. D. Steele

UNITED STATES DEPARTMENT OF THE INTERIOR GEOLOGICAL SURVEY - WATER RESOURCES DIVISION

Texas District Open-File Report

I.D. Yost, District Chief

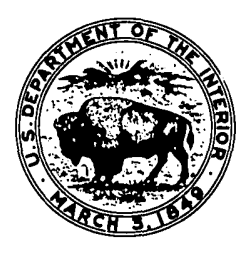


Abstract-1-

Introduction-

Available records-_- 4

Methods of evaluation- 7

Discussion of results- 10

Summary and conclusions- 51

References cited-1. 54 


\section{ILLUSTRATIONS}

Page

Figure 1. Map showing locations of water-quality stations used in the evaluation study-... 5

2. Flowchart showing procedures for data analysis and simulation-1.-.

3a. Graph showing dissolved solids versus discharge for station 08087300, Clear Ford Brazos River at Eliasville, Texas- 11

3b. Graph showing dissolved solids versus discharge for station 08111000, Navasota River near Bryan, Texas-- 12

4a. Graph showing silica versus specific conductance for station 08062500, Trinity River near Rosser, Texas-- 13

4b. Graph showing silica versus specific conductance for station 08176500, Guadalupe River at Victoria, Texas-_.

5a. Graph showing nitrate versus specific conductance for station 08062500, Trinity River near Rosser, Texas-- 15

5b. Graph showing phosphate versus specific conductance for station 08062500, Trinity River near Rosser, Texas-1-.

6a. Graph showing fluoride versus specific conductance for station 07342500, South Sulphur River near Cooper, Texas- 1 
Figure 6b. Graph showing $\mathrm{pH}$ versus specific conductance for station 07342500, South Sulphur River near Cooper, Texas-_. 18

7a. Graph showing dissolved solids versus specific conductance for station 08114000, Brazos River at Richmond, Texas- 34

7b. Graph showing dissolved solids versus specific conductance for station 08176500, Guadalupe River at Victoria, Texas

8a. Graph showing chloride versus specific conductance for station 08111000, Navasota River near Bryan, Texas- 36

8b. Graph showing chloride versus specific conductance for station 08147000, Colorado River near San Saba, Texas-

9. Graph showing residuals in milligrams per liter (actual minus simulated) for selected chemical constituents for the Brazos River at Richmond, Texas-

10. Graph showing residuals in milligrams per liter (actual minus simulated) for selected chemical constituents for the Guadalupe River at Victoria, Texas-- - 


\section{IILUSTRRATIONS- - Continued}

Page

Figure 1.1. Gaph showing annual discharge-weighted specific conductance values from composite samples versus

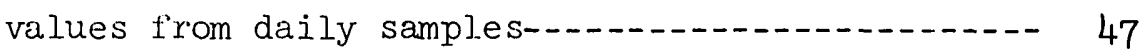

12. Graph showing actual and simulated dischargeweighted concentrations of calcium, chloride, and dissolved solids for Neches River at Evadale, $-\cdots$

Texas-

TABLES

Table 1. Summary of: the 12 chemical-quality sampling stations selected for the Texas study 6

2. Basic statistics of water-quality data sets from 12

Texas stations, 1962-66 water years-........... 19

3. Results of regression analyses, $c_{i}=a_{i}+b_{i} K_{S c}$, for selected chemical constituents for 12 Texas stations, 1962-66 water years-

4. Actual and simulated annual discharge-weighted concentrations for the Brazos River at Richmond, Texas, 1946-69 water years-_...

5. Actual and simulated annual discharge-weighted concentrations for the Guadalupe River at Victoria, Texas, 1946-69 water years-_...-. 
AN EVALUATION OF WATER-QUALITY RECORDS FOR TEXAS STREAMS

By

(r)

J. F. Blakey, R. O. Hawkinson, and T. D. Steele

U.S. Geological Survey

ABSTRACT

Chemical data for 12 streamflow-sampling stations in Texas, each hiving from 8 to 24 years of available historical. records, were analyzed to determine functional relationships between concentrations of the major inorganic constituents and specific conductance or stream discharge. Regression equations for each station were derived by using data for an identical 5-year period; the remaining record served as independent test data for checking the adequacy of the functions on the basis of continued specific-conductance determinations as an index variable.

Daily solute concentrations and loads at a sampling site were simulated, and monthly and annual averages were computed by using daily records of specific conductance in conjunction with the regression equations. Simulated annual mean concentrations of most individual solutes and dissolved solids averaged within 10-20 percent of comparable concentrations determined from the composited chemical analyses, although systematic biases were detected in results for some of the relationships used in the simulation. Results of the evaluation of the 12 stations indicated that regression equations, using specific conductance as the independent variable, can be used as an alternative to composite analyses for calculation of solute concentrations and loads in Texas streams and is a flexible system that can be applied with sufficient accuracy to satisfy most anticipated needs for data. 


\section{INTRODUC'IION}

Ever increasing eftorts to stop or reduce pollution and to develop water supplies result in a growing demand f'or current and applicable water-quality data. To provide this information, datacollection activities must be designed and periodically reviewed in relation to data requirements and uses.

For many years, the U.S. Geological Survey has placed major emphasis on collecting data on inorganic constituents. However, in the midsixties, the Survey began a shift in emphasis to organic-and biochemicaldata collection. This change was necessary to provide action agencies with information on environmental quality. In re-directing efforts, the Survey has reviewed and continues to review data-collection activities in relation to data-users' needs. Iimitations of funds and manpower, however, often require reducing one phase of the data-collection program in order to add others.

Statistical techniques in analysis of historical inorganic chemical-quality records are available to supplement judgment decisions for modifying data-collection activities. Large volumes of historical records can be analyzed with a sequence of computer programs to determine statistical properties of each constituent and, where applicable, to develop a regression relationship between each water-quality variable and streamflow or specific conductance. Additional computer programs in the sequence can be used to simulate water-quality records, to make time-trend analyses, and to regionalize water-quality conditions. 
'ind.s study has the following purposes:

(1.) T'o analyze, by statistical techniques, historical records of inorganic chemical quality of water at points on selected Texas streams;

(2) to present results from the computer-oriented analyses and to describe their usefulness as tools in data evaluation and network design; and

(3) to determine the accuracy with which inorganic chemical constituents can be estimated in a reduced data-collection program by using specific conductance or stream discharge as an index variable. 


\section{AVA TLABIEE RECOKDS}

Historical records of chemical quality for 12 Texas stations were selected for analysis. All 12 streams were operated as daily sampling stations during the 1960-69 water years, and they represent a crosssection of geologic and hydrologic conditions in Texas. Records of specilic conductance for periods of more than 2.0 years were available and were used in the analyses for four stations. Station information is summarized in table 1 and station locations are shown on figure 1 .

Figure 1 (caption on next page) belongs near here.

All chemical analyses in the data sets are for composited samples, except for a few discrete samples obtained during periods of high runoff. Composited samples consist of from two to 31. daily samples of "similar" specific conductance. Daily records of specific conductance and stream discharge were used in conjunction with the data sets for :tll stations 


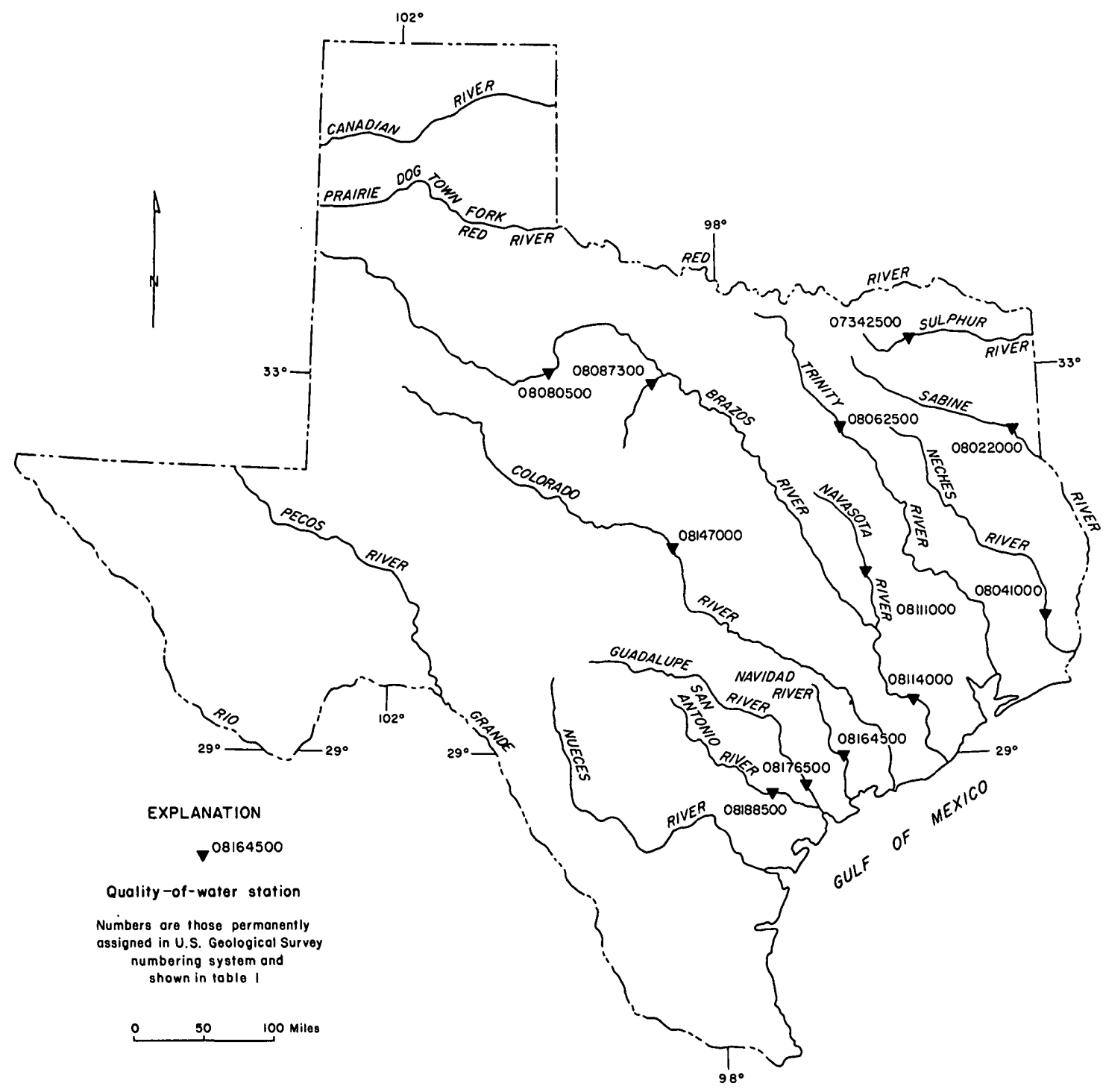

FIGURE I-Locations of water-quality stations used in the evaluation study 
Table 1.--Summary of the 12 chemical-quality sampling stations selected for the Texas study

\begin{tabular}{|c|c|c|c|c|c|}
\hline $\begin{array}{l}\text { USGS stätion } \\
\text { number }\end{array}$ & Location & $\begin{array}{l}\text { Drainage area } \\
\quad(\mathrm{sq} \cdot \mathrm{mi} .)\end{array}$ & $\begin{array}{c}\text { Period of record } 1 / \\
\text { (water years) }\end{array}$ & $\begin{array}{l}\text { Number } \\
\text { of years }\end{array}$ & $\begin{array}{l}\text { Number of } \\
\text { analyses } 2 /\end{array}$ \\
\hline 07342500 & South Sulphur River near Cooper & 527 & $1960-66,68-69$ & 9 & 194 \\
\hline 08022000 & Sabine River near Tatum & 3,586 & $1960-69$ & 10 & 190 \\
\hline 08041000 & Neches River at Evadale $3 /$ & 7,923 & $1948-69$ & 22 & 148 \\
\hline 08062500 & Trinity River near Rosser & 8,162 & $1960^{\circ}-69$ & 10 & 169 \\
\hline 08080500 & $\begin{array}{l}\text { Double Mountain Fork Brazos River } \\
\text { near Aspermont }\end{array}$ & 17,980 & $1960-69$ & 10 & 198 \\
\hline 08087300 & $\begin{array}{l}\text {-Clear Fork Brazos River at } \\
\text { Eliasville }\end{array}$ & 5,721 & $1962-69$ & 8 & 187 \\
\hline 08111000 & Navasota River near Bryan & 1,439 & $1960-69$ & 10 & 226 \\
\hline 08114000 & Brazos River at Richmond $3 /$ & 44,020 & $1946-69$ & 24 & 189 \\
\hline 08147000 & Colorado River near San Saba $3 /$ & 30,600 & $1948-69$ & 22 & 174 \\
\hline 08164500 & Navidad River near Ganado & 1,116 & $1960-69$ & 10 & 222 \\
\hline 08176500 & Guadalupe River at Victoria $3 /$ & 5,161 & $1946-69$ & 24 & 136 \\
\hline 08188500 & San Antonio River at Goliad & 3,918 & $1960-69$ & 10 & 186 \\
\hline Totals: & & & & 169 & 2,219 4 / \\
\hline
\end{tabular}

$1 /$ Period of record used for analysis, not necessarily including all data for the station.

2) Number of composited analyses for 1962-66 water years only, the period used for regression analysis.

3/ Selected for analyses of long-term records (20 years or longer).

4 A total of 4,515 composited analyses were available for 1960-69 water years. 


\section{METHODS OF EVALUATION}

IIistorical records of chemical analyses for the 12 stations were analyzed with a sequence of' computer programs developed by Steele (1970, 1971). A flow chart showing the procedures utilized in the data analysis and simulation is shown in figure 2 .

Figure 2 (caption on next page) belongs near here.

All inorganic chemical analyses of samples from the 12 stations for the 1960-69 period were retrieved from the Geological Survey's "Nationa $\perp$ water data storage and retrieval system," using the data set-up computer program. The basic statistics of the data set for each station were computed and printouts were examined to determine the number of data items and the range in concentration for each constituent in preparing for the graphic analysis. 

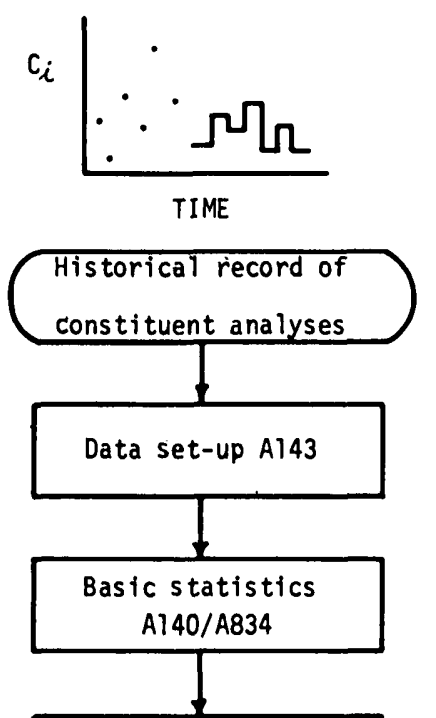

Graphic analysis A149

Regression analysis A137

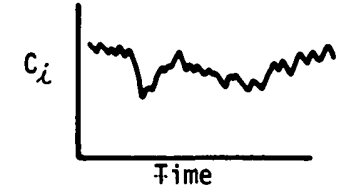

Daily chemical hydrographs
Alternative $B$

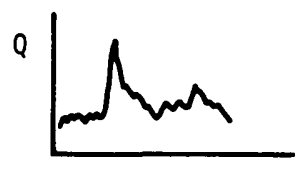

TIME

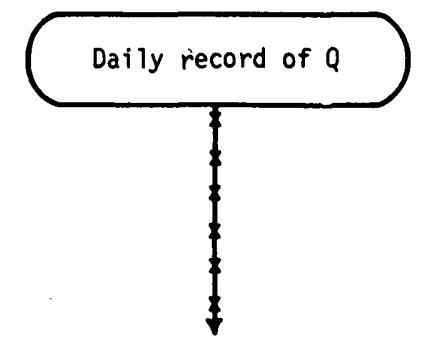

$K_{s c}=k Q^{n}$
Alternative $\mathrm{A}$

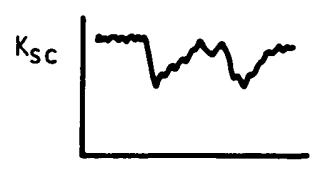

TIME

Daily record of $K_{S C}$

Weekly, monthly, and annual summaries

Time-trend analys is regionalization

Frequency-of-s ampling analys is A833

\section{Explanation}

$\mathrm{Ci}$ : Concentration of chemical constituent where $i=$ specific constituents.

$Q$ : Discharge.

$\mathrm{K}_{\mathrm{sc}}$ : Specific conductance.

$\mathrm{k}^{8 c}$ : Regression constant for $\log \mathrm{K}_{\mathrm{sc}}-\log \mathrm{Q}$ relationship.

$n$ : Regression coefficient for $\log K_{s c}-\log Q$ relationship.

ai : Regression constant for arithmetlc relationship between $K_{s c}$ and $c i$.

$\mathrm{bi}$ : Regression coefficient for arithmetic relationship between $\mathrm{K}_{\mathrm{sc}}$ and $\mathrm{C} i$.

(Adapted from Steele, 1970, p. 348)

Figure 2.--Flow chart showing procedures for data analysis and simulation. 
In the graphic analysis, all inorganic constituents $\left(C_{i}\right)$ were considered dependent variables and were plotted against discharge (Q), using logarithmic coordinates, and against specific conductance $\left(\mathrm{K}_{\mathrm{sc}}\right)$, using cartesian coordinates. The graphic display serves two important functions in the data evaluation: (1) Transcriptional errors and outliers are detected and checked; and (2) nonlinearity in a given dependent-independent variable relationship in the data set is readily detected. Anomalies in the data set can be corrected or omitted, and nonlinear relationships can be partitioned before the development of regression equations.

After examining the graphic analyses and making any necessary adjustments in each data set, regression equations were developed for $\mathrm{C}_{i}-\mathrm{K}_{\mathrm{sc}}$ relationships. Chemical analyses from identical 5-year periods (water years 1962-66) for each station were used as the calibration period for development of the relationships.

The regression equations were then used in conjunction with records of daily specific conductance and daily mean discharge to simulate inorganic-chemical data for the entire period of record available for each station (table 1). Records were simulated for a total of 169 station years, of which 109 years represented independent test data. 


\section{DISCUSSION OF RESLITS}

Graphic analyses of $C_{j}-Q\left(\right.$ and $K_{S C}-Q$ ) generally showed poor relationships, if any, between concentration and discharge for all stations. Typical plots of dissolved-solids concentration versus discharge for stations used in the study are shown on figures $3 a$ and $3 b$. These results

Figures $3 a$ and $3 b$ (captions on next page) belong near here.

coincide with the findings of many other investigators who have reported the poor correlation of $\mathrm{C}_{i}-\mathrm{Q}$ in streams in Texas and other western states (Rawson, 1967, p. 25; Colby, Hembree, and Rainwater, 1956, p. 123;

Leifeste, Blakey, and Hughes, 1971, p. 12; Steele, 1970, p. 349). On the other hand, $C_{i}-K_{S C}$ regression results were generally quite good, with high correlations and relatively low standard errors of estimate.

The basic statistics for all stations are summarized in table 2, and the regression equations for $\mathrm{C}_{i}-K_{S c}$ relationships are shown in table 3 . Also shown in table 3 is the range in $\mathrm{K}_{\mathrm{Sc}}$ over which the regression equations are applicable. Where negative intercept values $\left(a_{i}\right)$ resulted from fitting a straight line to slightly curvilinear $C_{i}-K_{s c}$ relationships, the lower limits of the range in $\mathrm{K}_{\mathrm{Sc}}$ have been adjusted upward to prevent calculation of negative concentrations. Constituents and variables in the tables are expressed in mg/l (milligrams per liter) except $Q$ (cubic feet per second), SC (micromhos per centimeter), and $\mathrm{pH}$ (units)--abbreviations are Q (stream discharge), HRD (hardness), NCH (non-carbonate hardness), DS (dissolved solids), and $\mathrm{SC}$ (specific conductance). Examples of $\mathrm{C}_{\mathrm{i}}-\mathrm{K}_{\mathrm{SC}}$ plotted data for 1962-66 water years used in the regression analysis are shown on figures $4 \mathrm{a}$ and $4 \mathrm{~b}$ to $6 \mathrm{a}$ and $6 \mathrm{~b}$.

Figures $4 a, 4 b, 5 a, 5 b, 6 a$, and $6 b$ (captions on next page) belong near here. 


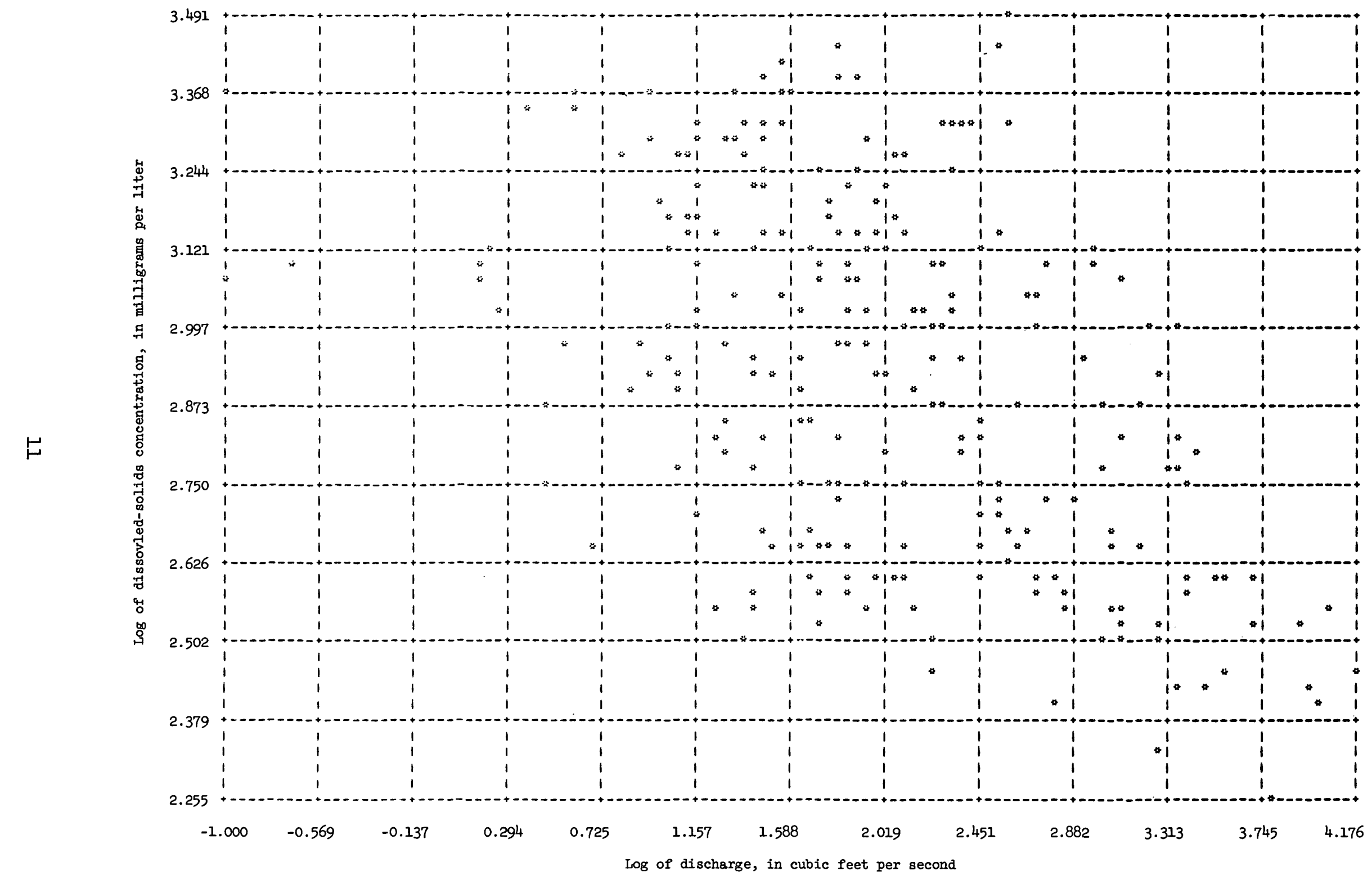

Figure 3a.--Dissolved solids versus discharge for station 08087300, Clear Fork Brazos River at Fliasville, Tex. 


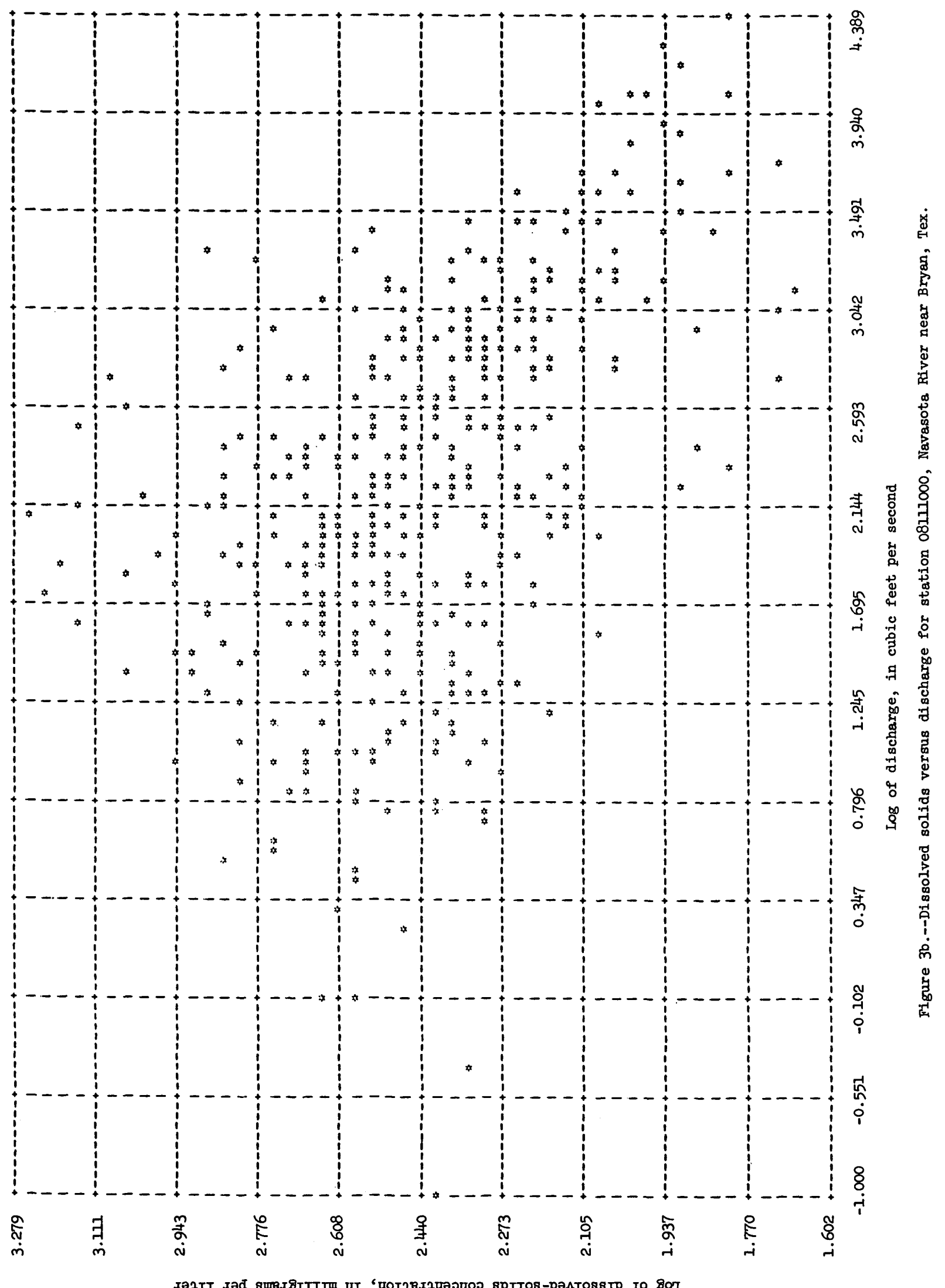

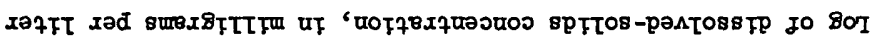




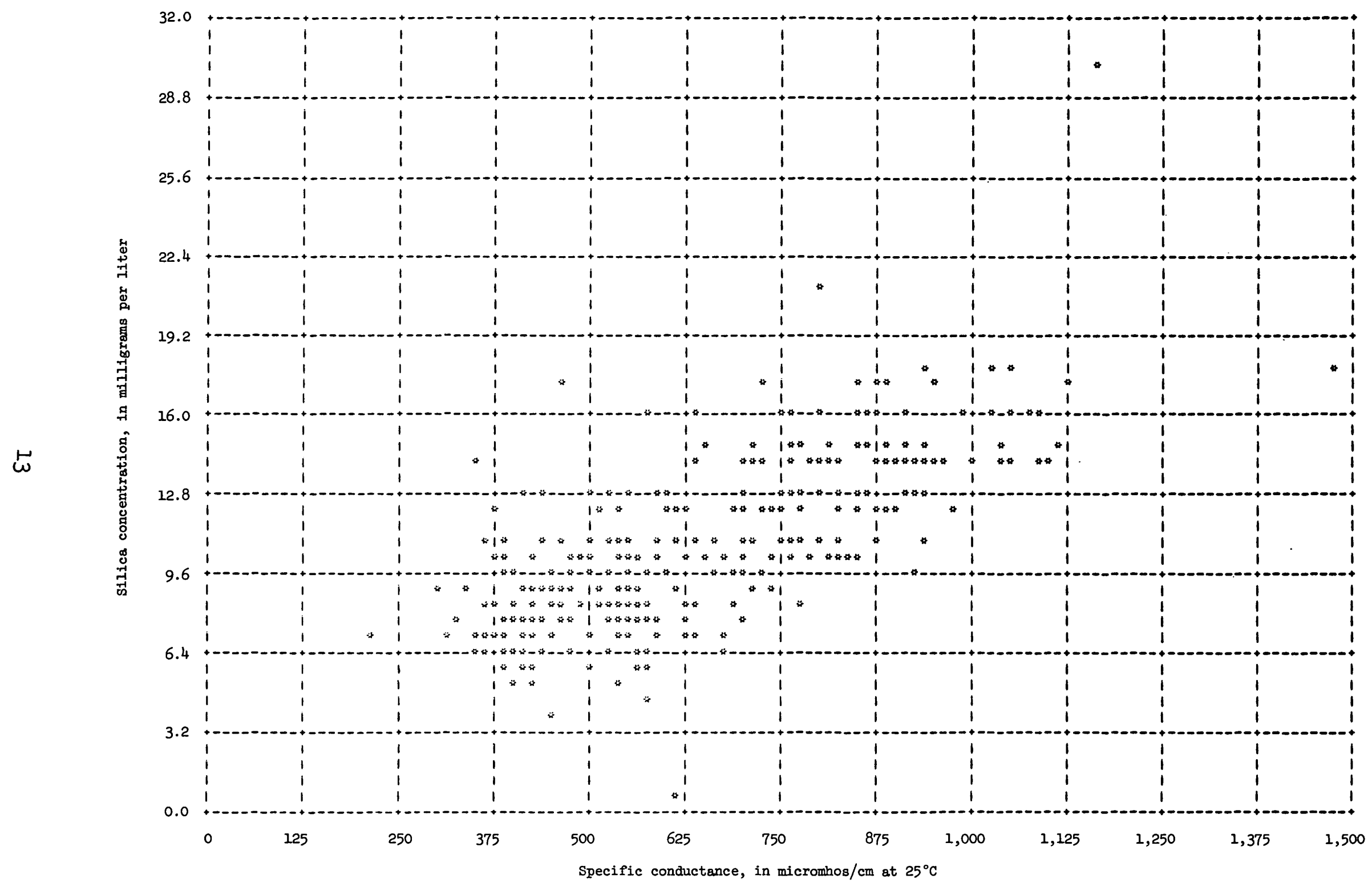

Figure 4a.--Silica versus specific conductance for station 08062500, Trinity River near Rosser, Tex. 


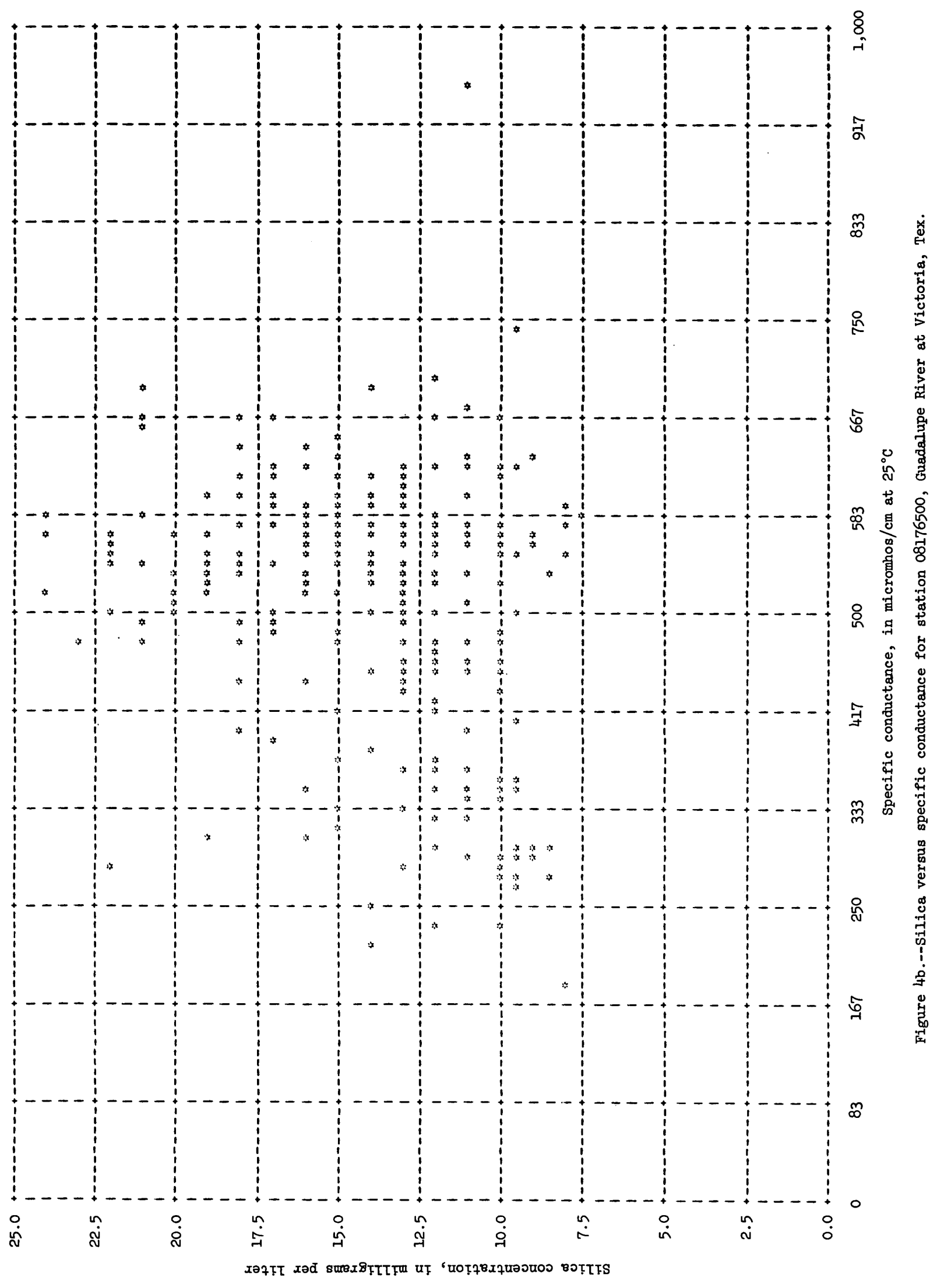




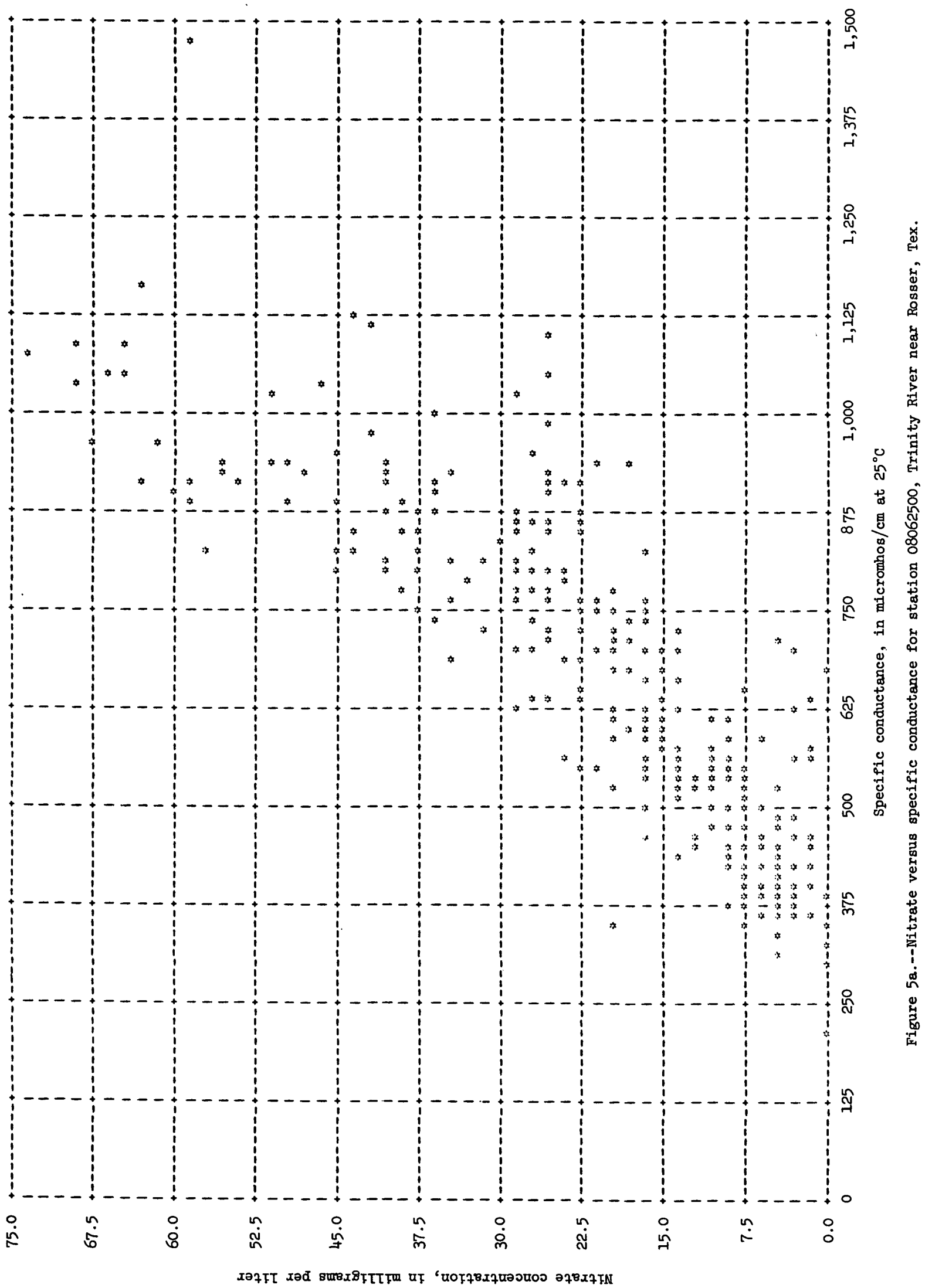




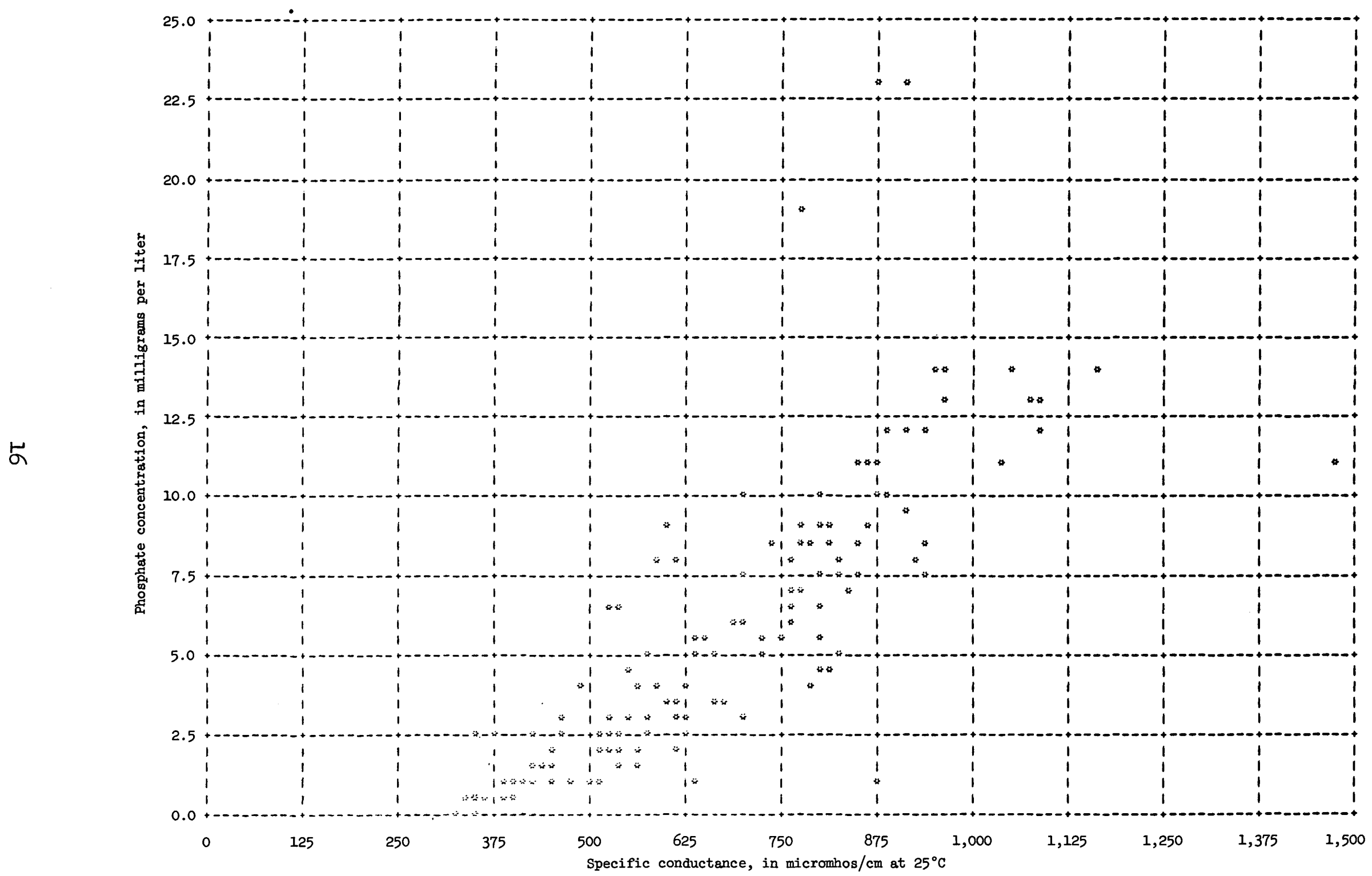

All stations have been processed

Figure 5b.--Phosphate versus specific conductance for station 08062500, Trinity River near Rosser, Tex. 


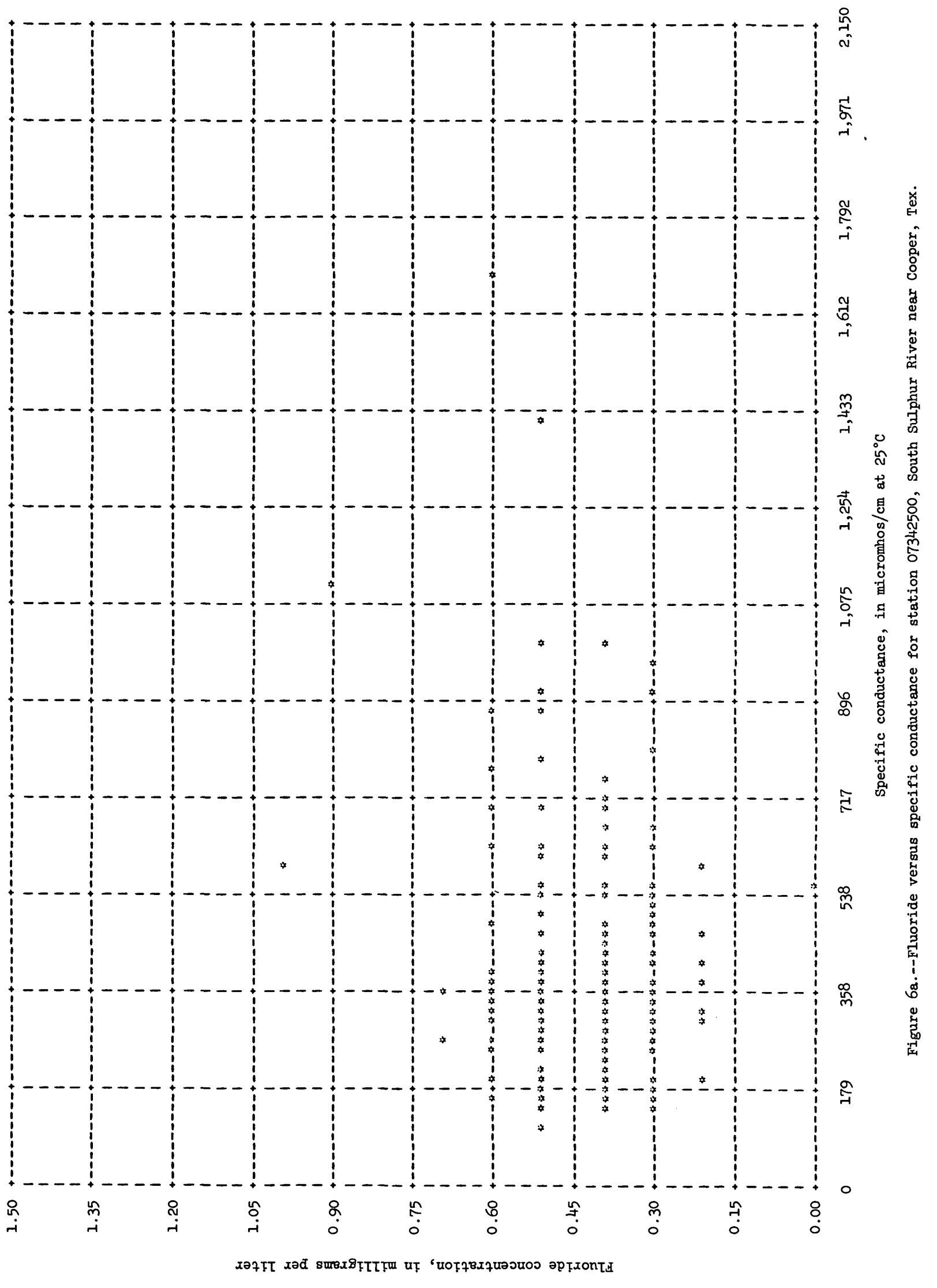




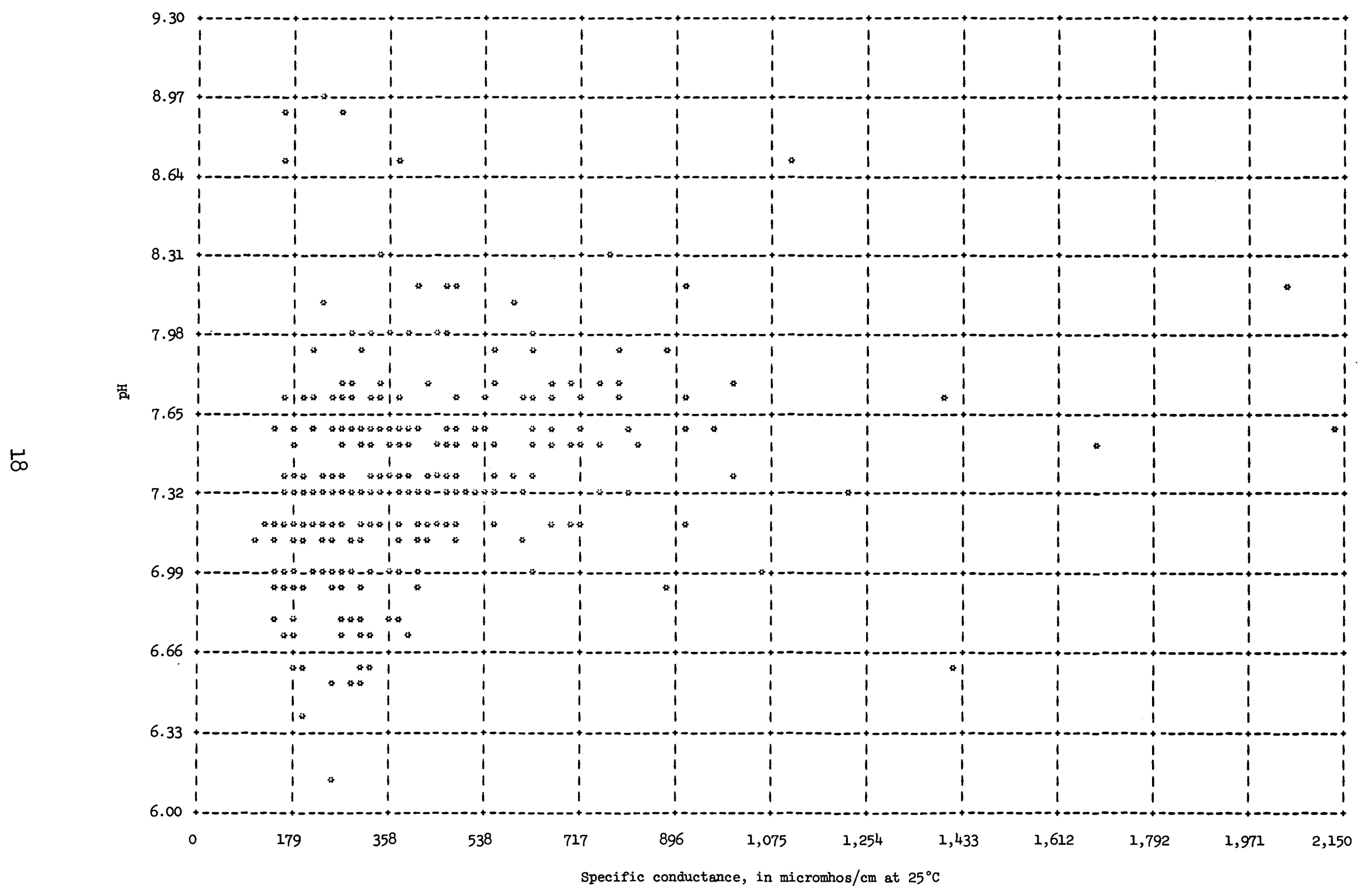

Figure 6b.--pH versus specific conductance for station 07342500 , South Sulphur River near Cooper, Tex. 
Table 2. Basic statistics of water-quality data sets from 12 Texas stations 1962-66 water years

(Constituents in milligrams per liter except as indicated)

$\begin{array}{llllll}\text { Constituent } & \text { Mean } & \begin{array}{l}\text { Standard } \\ \text { deviation }\end{array} & \begin{array}{c}\text { Sample } \\ \text { size }\end{array} & \begin{array}{l}\text { Maximum } \\ \text { observed }\end{array} & \begin{array}{l}\text { Minimum } \\ \text { observed }\end{array}\end{array}$ Skewness

07342500 SOUTH SULPHUR RIVER NEAR COOPER, TEX.

\begin{tabular}{|c|c|c|c|c|c|c|}
\hline a/ $Q$ & 707 & 2313 & 194 & 23,900 & 0.20 & 7.45 \\
\hline $\mathrm{SiO}_{2}$ & 10.8 & 3.48 & 175 & 22 & .0 & .19 \\
\hline $\mathrm{Ca}$ & 45.9 & 21.1 & 167 & 116 & 17 & 1.13 \\
\hline $\mathrm{Mg}$ & 5.02 & 2.64 & 167 & 14 & 1.1 & 1.18 \\
\hline $\mathrm{Na}$ & 35.8 & 38.8 & 175 & 326 & 4.4 & 3.94 \\
\hline $\mathrm{K}$ & 3.67 & .86 & 49 & 6.9 & 2.3 & 1.07 \\
\hline $\mathrm{HCO}_{3}$ & 155 & 72.2 & 194 & 403 & 43 & 1.01 \\
\hline $\mathrm{SO}_{4}$ & 35.3 & 22.4 & 194 & 111 & 8.2 & 1.74 \\
\hline $\mathrm{Cl}$ & 29.0 & 56.3 & 194 & 580 & 1.6 & 6.65 \\
\hline DS & 250 & 151 & 167 & 1,150 & 84 & 2.25 \\
\hline b/ $\mathrm{sC}$ & 406 & 254 & 194 & 2,130 & 124 & 2.65 \\
\hline HRD & 129 & 62.0 & 194 & 347 & 16 & 1.17 \\
\hline $\mathrm{NCH}$ & 8.73 & 18.8 & 194 & 153 & 0 & 5.03 \\
\hline F & .42 & .12 & 97 & .9 & .0 & .26 \\
\hline$c / \mathrm{pH}$ & 7.33 & .45 & 191 & 9.0 & 6.1 & .79 \\
\hline $\mathrm{NO}_{3}$ & 1.92 & 2.05 & 182 & 13 & .0 & 3.14 \\
\hline
\end{tabular}

08022000 SABINE RIVER NEAR TATUM, TEX.

\begin{tabular}{|c|c|c|c|c|c|c|}
\hline a/ $Q$ & 1590 & 3884 & 190 & 37,720 & 32 & 6.57 \\
\hline $\mathrm{SiO}_{2}$ & 12.7 & 3.78 & 186 & 19 & 1.1 & -.65 \\
\hline $\mathrm{Ca}$ & 16.2 & 4.88 & 186 & 34 & 5.2 & .64 \\
\hline $\mathrm{Mg}$ & 5.89 & 1.85 & 186 & 14 & 1.0 & .85 \\
\hline $\mathrm{Na}$ & 84.2 & 55.9 & 186 & 574 & 12 & 4.05 \\
\hline $\mathrm{K}$ & 3.72 & .66 & 42 & 5.6 & 2.1 & .43 \\
\hline $\mathrm{HCO}_{3}$ & 35.3 & 18.6 & 190 & 90 & 10 & .87 \\
\hline $\mathrm{SO}_{4}$ & 35.3 & 14.6 & 190 & 105 & 8.0 & 1.20 \\
\hline $\mathrm{Cl}$ & 128 & 84.7 & 190 & 880 & 18 & 4.13 \\
\hline DS & 303 & 156 & 188 & 1,610 & 61 & 3.48 \\
\hline $\mathrm{b} / \mathrm{sC}$ & 567 & 302 & 190 & 3,040 & 104 & 3.34 \\
\hline HRD & 64.1 & 18.3 & 190 & 124 & 15 & .39 \\
\hline $\mathrm{NCH}$ & 35.3 & 17.8 & 190 & 92 & 1 & .66 \\
\hline$F$ & .23 & .07 & 78 & .4 & .1 & .12 \\
\hline$c / \mathrm{pH}$ & 6.52 & .38 & 190 & 7.6 & 5.6 & .38 \\
\hline $\mathrm{NO}_{3}$ & 1.21 & 1.01 & 188 & 7.1 & .0 & 2.05 \\
\hline
\end{tabular}

See footnotes at end of table. 
Table 2. Basic statistics of water-quality data sets from 12 Texas stations 1962-66 water years--continued

\begin{tabular}{|c|c|c|c|c|c|c|}
\hline Constituent & Mean & $\begin{array}{l}\text { Standard } \\
\text { deviation }\end{array}$ & $\begin{array}{l}\text { Sample } \\
\text { size }\end{array}$ & $\begin{array}{l}\text { Maximum } \\
\text { observed }\end{array}$ & $\begin{array}{l}\text { Minimum } \\
\text { observed }\end{array}$ & Skewness \\
\hline \multicolumn{7}{|c|}{08041000 NECHES R } \\
\hline a/ $Q$ & 4176 & 4702 & 148 & 20,680 & 168 & 1.91 \\
\hline $\mathrm{SiO}_{2}$ & 12.8 & 3.22 & 148 & 22 & .0 & -.40 \\
\hline $\mathrm{Ca}$ & 9.30 & 2.50 & 148 & 16 & 2.5 & -.24 \\
\hline $\mathrm{Mg}$ & 3.29 & .97 & 148 & 6.3 & .0 & -.12 \\
\hline $\mathrm{Na}$ & 23.5 & 9.49 & 148 & 59 & 2.3 & .58 \\
\hline $\mathrm{K}$ & 2.81 & .79 & 37 & 3.8 & .9 & -.77 \\
\hline $\mathrm{HCO}_{3}$ & 29.4 & 13.0 & 147 & 72 & 6.0 & .64 \\
\hline $\mathrm{SO}_{4}$ & 18.8 & 6.15 & 147 & 34 & 2.8 & .49 \\
\hline $\mathrm{Cl}$ & 31.0 & 12.2 & 147 & 72 & 2.0 & .41 \\
\hline DS & 115 & 34.2 & 145 & 218 & 14 & -.02 \\
\hline b/ $\mathrm{sc}$ & 198 & 61.1 & 145 & 397 & 29 & .14 \\
\hline HRD & 36.8 & 9.67 & 145 & 61 & 6 & -.46 \\
\hline $\mathrm{NCH}$ & 12.4 & 6.38 & 145 & 28 & 0 & .60 \\
\hline $\mathrm{F}$ & .18 & .08 & 87 & .4 & .0 & -.32 \\
\hline$c / \mathrm{pH}$ & 6.53 & .45 & 145 & 8.6 & 5.7 & 1.06 \\
\hline $\mathrm{NO}_{3}$ & .69 & .42 & 145 & 3.8 & .0 & 2.96 \\
\hline $\mathrm{PO}_{4}$ & .04 & .03 & 17 & .08 & .00 & -.07 \\
\hline
\end{tabular}

08062500 TRINITY RIVER NEAR ROSSER, TEX.

$\begin{array}{lccrrrr}\text { a/ } & 2849 & 5412 & 169 & 42,000 & 235 & 4.34 \\ \mathrm{SiO}_{2} & 11.2 & 3.56 & 164 & 30 & .5 & .94 \\ \mathrm{Ca} & 53.7 & 8.60 & 165 & 73 & 21 & -.47 \\ \mathrm{Mg} & 5.35 & 1.89 & 165 & 14 & 1.6 & 1.20 \\ \mathrm{Na} & 76.6 & 43.2 & 164 & 253 & 13 & .66 \\ \mathrm{~K} & 7.99 & 3.33 & 51 & 17 & 3.8 & .63 \\ & & & & & & \\ \mathrm{HCO}_{3} & 157 & 22.6 & 169 & 211 & 58 & -.72 \\ \mathrm{SO}_{4} & 88.2 & 44.0 & 168 & 424 & 25 & 2.69 \\ \mathrm{Cl} & 56.6 & 28.2 & 169 & 120 & 7.8 & .21 \\ \mathrm{DS} & 403 & 140 & 166 & 975 & 112 & .50 \\ \mathrm{~b} / \mathrm{SC} & 668 & 215 & 169 & 1470 & 210 & .35 \\ \mathrm{HRD} & 156 & 22.5 & 169 & 200 & 64 & -.52 \\ \mathrm{NCH} & 26.5 & 14.5 & 169 & 98 & 0 & 1.00 \\ \mathrm{~F} & .74 & .35 & 82 & 2.3 & .2 & 1.35 \\ \mathrm{c} / \mathrm{PH} & 7.26 & .42 & 169 & 8.8 & 6.5 & .70 \\ \mathrm{NO}_{3} & 22.5 & 17.1 & 164 & 74 & .0 & .91 \\ \mathrm{PO}_{4} & 5.27 & 4.11 & 105 & 14 & .05 & .53\end{array}$

See footnotes at end of table. 
Table 2. Basic statistics of water-quality data sets from 12 Texas stations 1962-66 water years--continued

\begin{tabular}{|c|c|c|c|c|c|c|}
\hline Constituent & Mean & $\begin{array}{l}\text { Standard } \\
\text { deviation }\end{array}$ & $\begin{array}{l}\text { Sample } \\
\text { size }\end{array}$ & $\begin{array}{l}\text { Maximum } \\
\text { observed }\end{array}$ & $\begin{array}{l}\text { Minimum } \\
\text { observed }\end{array}$ & Skewness \\
\hline
\end{tabular}

08080500 DOUBLE MOUNTAIN FORK BRAZOS RIVER NEAR ASPERMONT, TEX.

\begin{tabular}{|c|c|c|c|c|c|c|}
\hline$a / Q$ & 225 & 730 & 198 & 7,250 & 0.10 & 6.43 \\
\hline $\mathrm{SiO}_{2}$ & 12.8 & 3.34 & 178 & 22 & .4 & -.11 \\
\hline $\mathrm{Ca}$ & 466 & 234 & 180 & 920 & 73 & .17 \\
\hline $\mathrm{Mg}$ & 74.1 & 49.6 & 179 & 209 & 8.8 & .61 \\
\hline $\mathrm{Na}$ & 596 & 355 & 183 & 1,360 & 33 & .15 \\
\hline K & 9.02 & 2.82 & 44 & 14 & 4.9 & .15 \\
\hline $\mathrm{HCO}_{3}$ & 103 & 22.3 & 195 & 176 & 61 & .49 \\
\hline $\mathrm{SO}_{4}$ & 1213 & 550 & 197 & 2,120 & 221 & .02 \\
\hline $\mathrm{Cl}$ & 976 & 657 & 198 & 2,300 & 58 & .25 \\
\hline DS & 3408 & 1814 & 182 & 6,470 & 599 & .09 \\
\hline b/ $/ \mathrm{sC}$ & 4713 & 2384 & 197 & 9,180 & 930 & .14 \\
\hline HRD & 1441 & 754 & 195 & 2,960 & 232 & .31 \\
\hline $\mathrm{NCH}$ & 1356 & 756 & 195 & 2,900 & 125 & .30 \\
\hline F & .65 & .29 & 43 & 1.7 & .3 & 1.62 \\
\hline$c / \mathrm{pH}$ & 7.30 & .29 & 194 & 7.9 & 6.3 & -.37 \\
\hline$-\mathrm{NO}_{3}$ & 1.69 & 1.36 & 176 & 10 & .0 & 1.77 \\
\hline
\end{tabular}

08087300 CLEAR FORK BRAZOS RIVER AT ELIASVILLE, TEX.

\begin{tabular}{|c|c|c|c|c|c|c|}
\hline$a / Q$ & 744 & 1842 & 187 & 12,100 & 0.10 & 4.19 \\
\hline$-\mathrm{SiO}_{2}$ & 7.03 & 3.06 & 173 & 20 & .4 & .82 \\
\hline $\mathrm{Ca}$ & 100 & 51.2 & 175 & 255 & 37 & 1.15 \\
\hline $\mathrm{Mg}$ & 33.5 & 25.4 & 175 & 159 & 4.4 & 1.57 \\
\hline $\mathrm{Na}$ & 191 & 140 & 173 & 627 & 20 & 1.05 \\
\hline K & 6.84 & 1.62 & 47 & 9.9 & 3.8 & -.13 \\
\hline $\mathrm{HCO}_{3}$ & 135 & 34.4 & 187 & 245 & 42 & .52 \\
\hline $\mathrm{SO}_{4}$ & 161 & 165 & 186 & 912 & 12 & 2.01 \\
\hline $\mathrm{Cl}$ & 366 & 278 & 187 & 1,420 & 42 & 1.29 \\
\hline DS & 947 & 618 & 177 & 3,020 & 180 & 1.05 \\
\hline $\mathrm{b} / \mathrm{sC}$ & 1639 & 1000 & 187 & 4,810 & 338 & 1.00 \\
\hline HRD & 384 & 226 & 187 & 1,290 & 110 & 1.31 \\
\hline $\mathrm{NCH}$ & 273 & 211 & 187 & 1,180 & 24 & 1.41 \\
\hline $\mathrm{F}$ & .30 & .08 & 92 & .5 & .0 & -.31 \\
\hline $\mathrm{c} / \mathrm{pH}$ & 7.33 & .32 & 187 & 8.7 & 6.5 & .47 \\
\hline $\mathrm{NO}_{3}$ & 2.26 & 1.28 & 173 & 7.6 & .0 & 1.00 \\
\hline
\end{tabular}

See footnotes at end of table. 
Table 2. Basic statistics of water-quality data sets from 12 Texas stations 1962-66 water years--continued

\begin{tabular}{|c|c|c|c|c|c|c|}
\hline Constituent & Mean & $\begin{array}{l}\text { Standard } \\
\text { deviation }\end{array}$ & $\begin{array}{c}\text { Sample } \\
\text { size }\end{array}$ & $\begin{array}{l}\text { Maximum } \\
\text { observed }\end{array}$ & $\begin{array}{l}\text { Minimum } \\
\text { observed }\end{array}$ & Skewness \\
\hline \multicolumn{7}{|c|}{08111000 NAVASOTA R } \\
\hline a/ $Q$ & 723 & 2469 & 226 & 24,100 & 0.10 & 6.69 \\
\hline $\mathrm{SiO}_{2}$ & 12.7 & 3.2 & 205 & 22 & 5.3 & .20 \\
\hline $\mathrm{Ca}$ & 29.2 & 14.6 & 206 & 82 & 5.5 & 1.04 \\
\hline $\mathrm{Mg}$ & 7.8 & 3.8 & 206 & 21 & 1.3 & .91 \\
\hline $\mathrm{Na}$ & 91.5 & 90.3 & 206 & 606 & 6.7 & 2.98 \\
\hline $\mathrm{K}$ & 4.09 & .64 & 50 & 5.7 & 2.6 & .23 \\
\hline $\mathrm{HCO}_{3}$ & 61.0 & 26.1 & 226 & 136 & 13 & .68 \\
\hline $\mathrm{SO}_{4}$ & 37.7 & 18.6 & 226 & 94 & 6.6 & .74 \\
\hline $\mathrm{Cl}$ & 156 & 160 & 226 & 1,020 & 4.8 & 2.72 \\
\hline DS & 374 & 276 & 224 & 1,810 & 49 & 2.33 \\
\hline b/ $\mathrm{sC}$ & 692 & 524 & 226 & 3,370 & 79 & 2.32 \\
\hline HRD & 107 & 53 & 226 & 288 & 19 & .95 \\
\hline $\mathrm{NCH}$ & 57 & 42 & 226 & 230 & 1.0 & 1.46 \\
\hline $\mathrm{F}$ & .27 & .10 & 88 & .5 & .0 & .08 \\
\hline c / $\mathrm{pH}$ & 6.94 & .32 & 225 & 7.7 & 6.3 & .34 \\
\hline $\mathrm{NO}_{3}$ & .92 & .74 & 204 & 4.2 & .0 & 1.66 \\
\hline
\end{tabular}

08114000 BRAZOS RIVER AT RICHMOND, TEX.

\begin{tabular}{lccrrrr}
\hline $\mathrm{a} / \mathrm{Q}$ & 7743 & 13159 & 189 & 90,580 & 312 & 3.90 \\
$\mathrm{SiO} 2$ & 10.4 & 2.62 & 183 & 18 & 4.4 & .69 \\
$\mathrm{Ca}$ & 62.6 & 16.1 & 182 & 107 & 32 & .37 \\
$\mathrm{Mg}$ & 11.1 & 4.49 & 182 & 25 & 3.3 & .66 \\
$\mathrm{Na}$ & 77.1 & 48.0 & 183 & 233 & 12 & 1.02 \\
$\mathrm{~K}$ & 3.91 & .59 & 35 & 5.3 & 2.6 & .26 \\
& & & & & & \\
$\mathrm{HCO}_{3}$ & 156 & 30.5 & 189 & 254 & 87 & .22 \\
$\mathrm{SO}_{4}$ & 77.3 & 42.1 & 189 & 224 & 21 & .97 \\
$\mathrm{Cl}$ & 111 & 73.8 & 189 & 362 & 8.7 & 1.06 \\
$\mathrm{DS}$ & 434 & 194 & 184 & 1,040 & 154 & .83 \\
$\mathrm{~b} / \mathrm{SC}$ & 754 & 328 & 189 & 1,830 & 257 & .86 \\
$\mathrm{HRD}$ & 200 & 57.7 & 189 & 370 & 97 & .45 \\
$\mathrm{NCH}$ & 72.0 & 44.9 & 189 & 227 & 10 & 1.05 \\
$\mathrm{~F}$ & .34 & .09 & 80 & .50 & .0 & -1.37 \\
$\mathrm{cH}$ & 7.4 & .52 & 189 & 8.1 & 6.8 & .49 \\
$\mathrm{pO}$ & 1.63 & 1.06 & 183 & 7.3 & .0 & 1.31 \\
$\mathrm{PO}_{4}$ & .11 & .08 & 25 & .29 & .00 & .33 \\
& & & & & & \\
\hline
\end{tabular}

See footnotes at end of table. 
Table 2. Basic statistics of water-quality data sets from 12 Texas stations 1962-66 water years--continued

\begin{tabular}{|c|c|c|c|c|c|c|}
\hline Constituent & Mean & $\begin{array}{l}\text { Standard } \\
\text { deviation }\end{array}$ & $\begin{array}{c}\text { Sample } \\
\text { size }\end{array}$ & $\begin{array}{l}\text { Maximum } \\
\text { observed }\end{array}$ & $\begin{array}{l}\text { Minimum } \\
\text { observed }\end{array}$ & Skewness \\
\hline & 081 & 7000 COLORADO & RIVER & NEAR SAN SABA, & TEX. & \\
\hline $\mathrm{a} / \mathrm{Q}$ & 1291 & 3077 & 174 & 26,410 & 0.10 & 6.01 \\
\hline $\mathrm{SiO}_{2}$ & 9.39 & 2.66 & 162 & 19 & 3.9 & 1.04 \\
\hline $\mathrm{Ca}$ & 63.0 & 20.5 & 162 & 202 & 32 & 2.83 \\
\hline $\mathrm{Mg}$ & 21.6 & 11.8 & 162 & 82 & 3.3 & 1.45 \\
\hline $\mathrm{Na}$ & 68.9 & 59.4 & 162 & 564 & 6.0 & 4.22 \\
\hline $\mathrm{K}$ & 4.14 & .89 & 41 & 7.4 & 2.6 & 1.51 \\
\hline $\mathrm{HCO}_{3}$ & 199 & 46.1 & 174 & 314 & 112 & .43 \\
\hline $\mathrm{SO}_{4}$ & 65.4 & 60.4 & 170 & 500 & 7.0 & 3.59 \\
\hline $\mathrm{Cl}$ & 126 & 119 & 174 & 1,000 & 3.4 & 3.44 \\
\hline DS & 460 & 270 & 164 & 2,440 & 149 & 3.29 \\
\hline $\mathrm{b} / \mathrm{sc}$ & 834 & 473 & 174 & 4,120 & 262 & 2.91 \\
\hline HRD & 249 & 95.1 & 174 & 842 & 102 & 2.24 \\
\hline $\mathrm{NCH}$ & 86.2 & 90.8 & 174 & 708 & 0 & 3.40 \\
\hline$F$ & .30 & .09 & 79 & .7 & .1 & .89 \\
\hline$c / \mathrm{pH}$ & 7.44 & .33 & 174 & 8.2 & 6.6 & 0 \\
\hline $\mathrm{NO}_{3}$ & 1.63 & 1.27 & 162 & 6.8 & .0 & 1.00 \\
\hline
\end{tabular}

08164500 NAVIDAD RIVER NEAR GANADO, TEX.

\begin{tabular}{|c|c|c|c|c|c|c|}
\hline$\stackrel{\mathrm{a} / \mathrm{Q}}{\mathrm{SiO}_{2}}$ & $\begin{array}{l}543 \\
19.0\end{array}$ & $\begin{array}{r}1040 \\
8.50\end{array}$ & $\begin{array}{l}222 \\
219\end{array}$ & $\begin{array}{r}7,079 \\
49\end{array}$ & $\begin{array}{l}3.5 \\
5.7\end{array}$ & $\begin{array}{l}3.77 \\
1.12\end{array}$ \\
\hline $\mathrm{Ca}$ & 43.6 & 21.7 & 212 & 100 & 8.5 & .51 \\
\hline $\mathrm{Mg}$ & 6.27 & 3.69 & 212 & 21 & 1.4 & 1.44 \\
\hline $\mathrm{Na}$ & 39.7 & 22.5 & 217 & 138 & 3.0 & .91 \\
\hline $\mathrm{K}$ & 3.51 & .94 & 67 & 6.4 & 2.4 & 1.61 \\
\hline $\mathrm{HCO}_{3}$ & $157^{\circ}$ & 72.6 & 218 & 326 & 34 & .24 \\
\hline $\mathrm{SO}_{4}$ & 14.8 & 5.74 & 222 & 37 & 4.0 & .82 \\
\hline $\mathrm{Cl}$ & 53.6 & 30.9 & 222 & 170 & 3.8 & .60 \\
\hline DS & 256 & 118 & 221 & 614 & 63 & .27 \\
\hline$\underline{b} / \mathrm{sC}$ & 448 & 201 & 222 & 1,020 & 106 & .18 \\
\hline HRD & 134 & 61.5 & 218 & 274 & 26 & .23 \\
\hline $\mathrm{NCH}$ & 6.46 & 7.96 & 218 & 57 & 0 & 2.59 \\
\hline$F$ & .34 & .11 & 116 & .7 & .1 & .29 \\
\hline $\mathrm{c} / \mathrm{pH}$ & 7.35 & .39 & 216 & 8.5 & 6.2 & -.23 \\
\hline $\mathrm{NO}_{3}$ & .95 & .74 & 219 & 3.5 & .0 & 1.04 \\
\hline $\mathrm{PO}_{4}$ & .06 & .07 & 29 & .34 & .00 & 2.72 \\
\hline
\end{tabular}

See footnotes at end of table. 
Table 2. Basic statistics of water-quality data sets from 12 Texas stations 1962-66 water years--continued

\begin{tabular}{|c|c|c|c|c|c|c|}
\hline Constituent & Mean & $\begin{array}{l}\text { Standard } \\
\text { deviation }\end{array}$ & $\begin{array}{l}\text { Sample } \\
\text { size }\end{array}$ & $\begin{array}{l}\text { Maximum } \\
\text { observed }\end{array}$ & $\begin{array}{l}\text { Minimum } \\
\text { observed }\end{array}$ & Skewness \\
\hline \multicolumn{7}{|c|}{08176500 GUADALUPE $R$} \\
\hline $\mathrm{a} / \mathrm{Q}$ & 1597 & 1941 & 136 & 10,360 & 165 & 2.57 \\
\hline $\mathrm{SiO}_{2}$ & 13.8 & 3.62 & 134 & 24 & 7.3 & .58 \\
\hline $\mathrm{Ca}$ & 54.6 & 10.8 & 133 & 80 & 27 & -.10 \\
\hline $\mathrm{Mg}$ & 13.7 & 4.52 & 132 & 25 & 2.9 & -.59 \\
\hline $\mathrm{Na}$ & 28.7 & 8.98 & 134 & 53 & 5.3 & .02 \\
\hline $\mathrm{K}$ & 3.01 & .87 & 33 & 5.6 & 2.1 & 1.43 \\
\hline $\mathrm{HCO}_{3}$ & 202 & 56.4 & 136 & 282 & 107 & -- \\
\hline $\mathrm{SO}_{4}$ & 28.4 & 6.98 & 134 & 46 & 7.4 & -.07 \\
\hline $\mathrm{Cl}$ & 38.6 & 12.9 & 134 & 78 & 5.7 & .06 \\
\hline DS & 286 & 61.1 & 133 & 432 & 133 & -.69 \\
\hline $\mathrm{b} / \mathrm{sc}$ & 493 & 101 & 136 & 695 & 217 & -.78 \\
\hline HRD & 192 & 41.0 & 134 & 272 & 90 & -.60 \\
\hline $\mathrm{NCH}$ & 22.9 & 10.9 & 134 & 55 & 0 & .14 \\
\hline F & .30 & .07 & 79 & .5 & .1 & -.05 \\
\hline c $/ \mathrm{pH}$ & 7.54 & .31 & 135 & 8.4 & 6.6 & .26 \\
\hline $\mathrm{NO}_{3}$ & 2.58 & 1.15 & 135 & 5.8 & .0 & .16 \\
\hline
\end{tabular}

08188500 SAN ANTONIO RIVER AT GOLIAD, TEX.

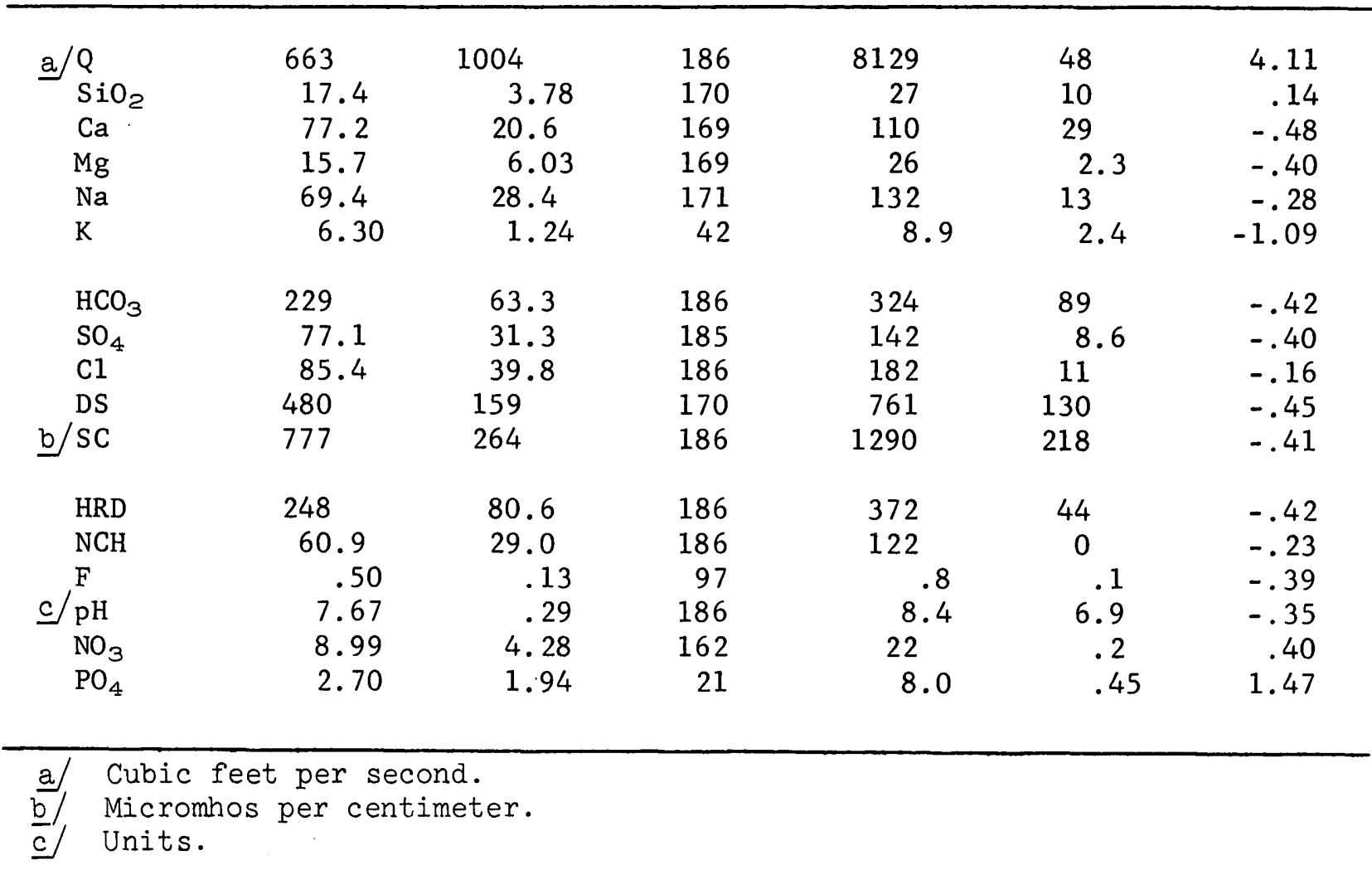


Table 3. Results of regression analyses, $C_{i}=a_{i}+b_{i} K_{s c}$, for selected chemical constituents for 12 Texas stations, 1962-66 water years

\begin{tabular}{|c|c|c|c|c|c|c|c|c|c|}
\hline $\begin{array}{l}\text { Variable } \\
\text { (i) }\end{array}$ & $\begin{array}{l}\text { No. of } \\
\text { paired } \\
\text { values }\end{array}$ & $\begin{array}{c}\text { Intercept } \\
\left(a_{i}\right)\end{array}$ & $\begin{array}{l}\text { Regression } \\
\text { coefficient } \\
\quad\left(b_{i}\right)\end{array}$ & $\begin{array}{c}\text { Mean } \\
\mathrm{C}_{i}\end{array}$ & $\begin{array}{l}\text { Standard } \\
\text { deviation } \\
\mathrm{C}_{i}\end{array}$ & $\begin{array}{l}\text { Correlation } \\
\text { coefficient } \\
(\mathrm{r})\end{array}$ & $\begin{array}{l}\text { Std. error } \\
\text { estimate } \\
\left(\mathrm{SE}_{\mathrm{e}}\right)\end{array}$ & $\begin{array}{l}\mathrm{SE}_{\mathrm{e}} \text { as } \\
\text { percent } \\
\text { of mean }\end{array}$ & Range in $\mathrm{K}_{\mathrm{SC}}$ \\
\hline
\end{tabular}

07342500 SOUTH SULPHUR RIVER NEAR COOPER, TEX.

\begin{tabular}{|c|c|c|c|c|c|c|c|c|c|c|}
\hline $\mathrm{Ca}$ & 167 & 18.27 & 0.0651 & 45.9 & 21.1 & 0.80 & 12.6 & 27 & 110 & -2200 \\
\hline $\mathrm{Mg}$ & 167 & 1.73 & .0078 & 5.02 & 2.64 & .77 & 1.69 & 34 & 110 & -2200 \\
\hline $\mathrm{Na}$ & 175 & -22.66 & .1408 & 35.8 & 38.8 & .94 & 13.6 & 38 & 170 & -2200 \\
\hline $\mathrm{K}$ & 49 & 2.66 & .0028 & 3.67 & .86 & .61 & .69 & 19 & 110 & -2200 \\
\hline $\mathrm{HCO}_{3}$ & 194 & 70.67 & .2072 & 155 & 72.2 & .73 & 49.6 & 32 & 110 & -2200 \\
\hline $\mathrm{SO}_{4}$ & 194 & 9.79 & .0628 & 35.3 & 22.4 & .71 & 15.8 & 45 & 110 & -2200 \\
\hline $\mathrm{C} 1$ & 194 & -46.78 & .1867 & 29.0 & 56.3 & .84 & 30.4 & 105 & 255 & -2200 \\
\hline DS & 167 & 8.37 & .5723 & 250 & 151 & .99 & 17.1 & 7 & 110 & -2200 \\
\hline HRD & 194 & 48.78 & . 1981 & 129 & 62.0 & .81 & 36.3 & 28 & 110 & -2200 \\
\hline
\end{tabular}

U

08022000 SABINE RIVER NEAR TATUM, TEX.

\begin{tabular}{|c|c|c|c|c|c|c|c|c|c|c|}
\hline $\mathrm{Ca}$ & 185 & 9.01 & 0.0127 & 16.1 & 4.71 & 0.65 & 3.60 & 22 & 100 & -3100 \\
\hline $\mathrm{Mg}$ & 185 & 3.14 & .0049 & 5.87 & 1.84 & .64 & 1.41 & 24 & 100 & -3100 \\
\hline $\mathrm{Na}$ & 186 & -20.83 & .1838 & 84.2 & 55.9 & .99 & 6.81 & 8 & 115 & -3100 \\
\hline $\mathrm{K}$ & 41 & 2.93 & .0013 & 3.70 & .65 & .46 & .58 & 16 & 100 & -3100 \\
\hline $\mathrm{HCO}_{3}$ & 190 & 24.77 & .0185 & 35.3 & 18.6 & .29 & 17.8 & 50 & 100 & -3100 \\
\hline $\mathrm{SO}_{4}$ & 190 & 18.09 & .0304 & 35.3 & 14.6 & .63 & 11.3 & 32 & 100 & -3100 \\
\hline $\mathrm{C} 1$ & 190 & -29.78 & .2776 & 128 & 84.7 & .99 & 12.8 & 10 & 110 & -3100 \\
\hline DS & 188 & 9.99 & .5154 & 303 & 156 & .99 & 10.1 & 3 & 100 & -3100 \\
\hline HRD & 190 & 39.85 & .0428 & 64.1 & 18.3 & .70 & 13.1 & 20 & 100 & -3100 \\
\hline
\end{tabular}


Table 3. Results of regression analyses, $c_{i}=a_{i}+b_{i} k_{s c}$, for selected chemical constituents for 12 Texas stations, 1962-66 water years--Continued

\begin{tabular}{|c|c|c|c|c|c|c|c|c|c|}
\hline $\begin{array}{l}\text { Variable } \\
\text { (i) }\end{array}$ & $\begin{array}{l}\text { No. of } \\
\text { paired } \\
\text { values }\end{array}$ & $\begin{array}{c}\text { Intercept } \\
\left(a_{i}\right)\end{array}$ & $\begin{array}{l}\text { Regression } \\
\text { coefficient } \\
\left(b_{i}\right)\end{array}$ & $\begin{array}{c}\text { Mean } \\
C_{i}\end{array}$ & $\begin{array}{c}\text { Standard } \\
\text { deviation } \\
\mathrm{C}_{i}\end{array}$ & $\begin{array}{l}\text { Correlation } \\
\text { coefficient } \\
\text { (r) }\end{array}$ & $\begin{array}{l}\text { Std. error } \\
\text { estimate } \\
\left(\mathrm{SE}_{\mathrm{e}}\right)\end{array}$ & $\begin{array}{l}\mathrm{SE} \text { as } \\
\text { percent } \\
\text { of mean }\end{array}$ & Range in $\mathrm{K}_{\mathrm{sC}}$ \\
\hline \multicolumn{10}{|c|}{08041000 NECHES RIVER AT EVADALE, TEX. } \\
\hline $\begin{array}{l}\mathrm{Ca} \\
\mathrm{Mg} \\
\mathrm{Na} \\
\mathrm{K}\end{array}$ & $\begin{array}{r}145 \\
145 \\
145 \\
35\end{array}$ & $\begin{array}{r}2.21 \\
.98 \\
-5.98 \\
1 \quad 1.64\end{array}$ & $\begin{array}{r}0.0358 \\
.0117 \\
.1488 \\
.0068\end{array}$ & $\begin{array}{c}9.30 \\
3.30 \\
23.5 \\
2.81\end{array}$ & $\begin{array}{r}2.49 \\
.97 \\
9.43 \\
.81\end{array}$ & $\begin{array}{r}0.88 \\
.73 \\
.96 \\
.53\end{array}$ & $\begin{array}{r}1.20 \\
.66 \\
2.48 \\
.69\end{array}$ & $\begin{array}{l}13 \\
20 \\
11 \\
25\end{array}$ & $\begin{array}{l}25-410 \\
25-410 \\
42-410 \\
25-410\end{array}$ \\
\hline $\begin{array}{l}\mathrm{HCO}_{3} \\
\mathrm{SO}_{4} \\
\mathrm{Cl} \\
\mathrm{DS} \\
\mathrm{HRD}\end{array}$ & $\begin{array}{l}145 \\
145 \\
145 \\
145 \\
145\end{array}$ & $\begin{array}{r}-2.59 \\
9.25 \\
-7.70 \\
5.67 \\
9.34\end{array}$ & $\begin{array}{l}.1627 \\
.0481 \\
.1960 \\
.5534 \\
.1384\end{array}$ & $\begin{array}{c}29.7 \\
18.8 \\
31.1 \\
115 \\
36.8\end{array}$ & $\begin{array}{c}13.0 \\
6.18 \\
12.3 \\
34.2 \\
9.67\end{array}$ & $\begin{array}{l}.76 \\
.47 \\
.98 \\
.99 \\
.87\end{array}$ & $\begin{array}{l}8.36 \\
5.46 \\
2.59 \\
5.22 \\
4.69\end{array}$ & $\begin{array}{r}28 \\
29 \\
8 \\
5 \\
13\end{array}$ & $\begin{array}{l}25-410 \\
25-410 \\
42-410 \\
25-410 \\
25-410\end{array}$ \\
\hline \multicolumn{10}{|c|}{08062500} \\
\hline $\begin{array}{l}\mathrm{SiO}_{2} \\
\mathrm{Ca} \\
\mathrm{Mg} \\
\mathrm{Na} \\
\mathrm{K}\end{array}$ & $\begin{array}{r}164 \\
165 \\
165 \\
164 \\
51\end{array}$ & $\begin{array}{r}3.77 \\
52.27 \\
1.09 \\
-55.12 \\
-2.14\end{array}$ & $\begin{array}{r}0.0110 \\
.0022 \\
.0064 \\
.1964 \\
.0149\end{array}$ & $\begin{array}{l}11.2 \\
53.7 \\
5.35 \\
76.6 \\
7.99\end{array}$ & $\begin{array}{c}3.56 \\
8.60 \\
1.89 \\
43.2 \\
3.33\end{array}$ & $\begin{array}{r}0.66 \\
-.05 \\
.72 \\
.98 \\
.93\end{array}$ & $\begin{array}{l}2.67 \\
8.61 \\
1.32 \\
8.58 \\
1.25\end{array}$ & $\begin{array}{l}24 \\
16 \\
25 \\
11 \\
16\end{array}$ & $\begin{array}{r}200-1500 \\
200-1500 \\
200-1500 \\
290-1500 \\
200-1500\end{array}$ \\
\hline $\begin{array}{l}\mathrm{HCO}_{3} \\
\mathrm{SO}_{4} \\
\mathrm{Cl} \\
\mathrm{DS} \\
\mathrm{HRD} \\
\mathrm{NO}_{3} \\
\mathrm{PO}_{4}\end{array}$ & $\begin{array}{l}169 \\
167 \\
168 \\
166 \\
169 \\
164 \\
105\end{array}$ & $\begin{array}{l}144 \\
-25.06 \\
-31.08 \\
-25.29 \\
132 \\
-25.60 \\
-5.93\end{array}$ & $\begin{array}{l}.0205 \\
.1674 \\
.1319 \\
.6412 \\
.0350 \\
.0717 \\
.0164\end{array}$ & $\begin{array}{c}157 \\
86.1 \\
56.4 \\
403 \\
156 \\
22.5 \\
5.27\end{array}$ & $\begin{array}{c}22.6 \\
35.5 \\
28.1 \\
140 \\
22.5 \\
17.1 \\
4.11\end{array}$ & $\begin{array}{l}.18 \\
.97 \\
.97 \\
.99 \\
.33 \\
.90 \\
.90\end{array}$ & $\begin{array}{c}22.2 \\
8.24 \\
6.71 \\
15.6 \\
21.3 \\
7.36 \\
1.79\end{array}$ & $\begin{array}{r}14 \\
10 \\
12 \\
4 \\
14 \\
33 \\
34\end{array}$ & $\begin{array}{l}200-1500 \\
200-1300 \\
290-1400 \\
200-1500 \\
200-1500 \\
370-1500 \\
370-1500\end{array}$ \\
\hline
\end{tabular}


Table 3. Results of regression analyses, $c_{i}=a_{i}+b_{i} k_{s c}$, for selected chemical constituents for 12 Texas stations, 1962-66 water years--Continued

\begin{tabular}{|c|c|c|c|c|c|c|c|c|c|c|}
\hline $\begin{array}{l}\text { Variable } \\
\text { (i) }\end{array}$ & $\begin{array}{l}\text { No. of } \\
\text { paired } \\
\text { values }\end{array}$ & $\begin{array}{c}\text { Intercept } \\
\qquad\left(\mathrm{a}_{i}\right)\end{array}$ & $\begin{array}{l}\text { Regression } \\
\text { coefficient } \\
\qquad\left(b_{i}\right)\end{array}$ & $\begin{array}{c}\text { Mean } \\
\mathrm{C}_{i}\end{array}$ & $\begin{array}{l}\text { Standard } \\
\text { deviation } \\
C_{i}\end{array}$ & $\begin{array}{l}\text { Correlation } \\
\text { coefficient } \\
(\mathrm{r})\end{array}$ & $\begin{array}{c}\text { Std. error } \\
\text { estimate } \\
\left(\mathrm{SE}_{\mathrm{e}}\right)\end{array}$ & $\begin{array}{l}S E_{e} \text { as } \\
\text { percent } \\
\text { of mean }\end{array}$ & Range & in $\mathrm{K}_{\mathrm{SC}}$ \\
\hline \multicolumn{11}{|c|}{08080500 DOUBLE MOUNTAIN FORK BRAZOS } \\
\hline $\mathrm{Ca}$ & 180 & 35.32 & 0.0894 & 466 & 234 & 0.93 & 86.9 & 19 & 900 & -9200 \\
\hline $\mathrm{Mg}$ & 179 & -15.98 & .0187 & 74.1 & 49.6 & .92 & 19.8 & 27 & 900 & -9200 \\
\hline $\mathrm{Na}$ & 183 & -87.58 & .1427 & 596 & 355 & .97 & 81.5 & 14 & 900 & -9200 \\
\hline $\mathrm{K}$ & 44 & 4.45 & .0009 & 9.02 & 2.82 & .88 & 1.36 & 15 & 900 & -9200 \\
\hline $\mathrm{HCO}_{3}$ & 195 & 99.18 & .0009 & 103 & 22.3 & .06 & 22.2 & 22 & 900 & -9200 \\
\hline $\mathrm{SO}_{4}$ & 196 & 195 & .2150 & 1210 & 549 & .93 & 196 & 16 & 900 & -9200 \\
\hline $\mathrm{Cl}$ & 197 & -316 & .2731 & 971 & 656 & .99 & 81.1 & 8 & 1200 & -9200 \\
\hline $\mathrm{DS}$ & 182 & -119 & .7381 & 3410 & 1810 & .99 & 212 & 6 & 900 & -9200 \\
\hline HRD & 195 & 46.99 & .2960 & 1440 & 754 & .94 & 267 & 19 & 900 & -9200 \\
\hline \multicolumn{11}{|c|}{08087300 CLEAR } \\
\hline $\mathrm{Ca}$ & 175 & 19.99 & 0.0488 & 100 & 51.4 & 0.96 & 14.3 & 14 & 300 & -4850 \\
\hline $\mathrm{Mg}$ & 175 & -5.18 & .0235 & 33.5 & 25.5 & .93 & 9.39 & 28 & 300 & -4850 \\
\hline $\mathrm{Na}$ & 173 & -36.26 & .1375 & 191 & 140 & .99 & 16.8 & 9 & 300 & -4850 \\
\hline $\mathrm{K}$ & 47 & 4.77 & .0013 & 6.84 & 1.63 & .78 & 1.03 & 15 & 300 & -4850 \\
\hline $\mathrm{HCO}_{3}$ & 187 & 111 & .0145 & 135 & 34.5 & .42 & 31.3 & 23 & 300 & -4850 \\
\hline $\mathrm{SO}_{4}$ & 186 & -35.08 & .1198 & 161 & 165 & .72 & 114 & 71 & 300 & -4850 \\
\hline $\mathrm{C} 1$ & 187 & -74.29 & .2689 & 366 & 278 & .97 & 70.0 & 19 & 300 & -4850 \\
\hline DS & 177 & -53.75 & .6031 & 947 & 619 & .99 & 77.6 & 8 & 300 & -4850 \\
\hline HRD & 187 & 30.17 & .2161 & 384 & 227 & .96 & 66.7 & 17 & 300 & -4850 \\
\hline
\end{tabular}


Table 3. Results of regression analyses, $C_{i}=a+b_{i} K_{s c}$, for selected chemical constituents for 12 Texas stations, 1962-66 water year--Continued

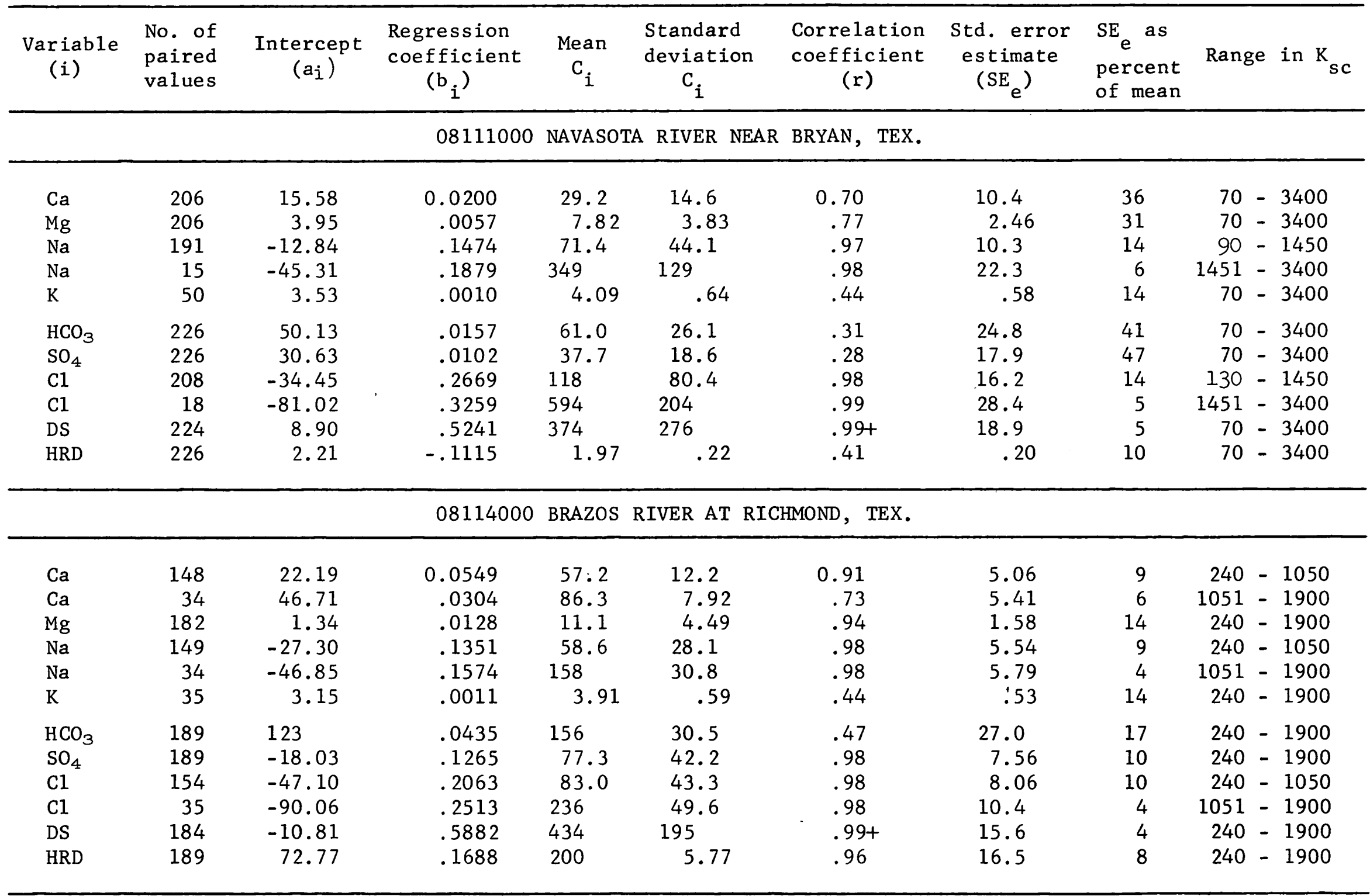


Table 3. Results of regression analyses, $c_{i}=a_{i}+b_{i} K_{s c}$, for selected chemical constituents for 12 Texas stations, 1962-66 water years--Continued

\begin{tabular}{|c|c|c|c|c|c|c|c|c|c|}
\hline $\begin{array}{l}\text { Variable } \\
\text { (i) }\end{array}$ & $\begin{array}{l}\text { No. of } \\
\text { paired } \\
\text { values }\end{array}$ & $\begin{array}{l}\text { Intercept } \\
\qquad\left(a_{i}\right)\end{array}$ & $\begin{array}{l}\text { Regression } \\
\text { coefficient } \\
\left(\mathrm{b}_{i}\right)\end{array}$ & $\begin{array}{c}\text { Mean } \\
\mathrm{C}_{i}\end{array}$ & $\begin{array}{c}\text { Standard } \\
\text { deviation } \\
\mathrm{C}_{i}\end{array}$ & $\begin{array}{c}\text { Correlation } \\
\text { coefficient } \\
(r)\end{array}$ & $\begin{array}{l}\text { Std. error } \\
\text { estimate } \\
\left(\mathrm{SE}_{\mathrm{e}}\right)\end{array}$ & $\begin{array}{l}\mathrm{SE}_{e} \text { as } \\
\text { percent } \\
\text { of mean }\end{array}$ & Range in $\mathrm{K}_{\mathrm{SC}}$ \\
\hline \multicolumn{10}{|c|}{08147000 COLORADO RIVER NEAR SAN SABA, TEX. } \\
\hline $\begin{array}{l}\mathrm{Ca} \\
\mathrm{Mg} \\
\mathrm{Na} \\
\mathrm{K}\end{array}$ & $\begin{array}{r}162 \\
162 \\
162 \\
41\end{array}$ & $\begin{array}{r}28.30 \\
3.99 \\
-37.45 \\
3.04\end{array}$ & $\begin{array}{r}0.0433 \\
.0219 \\
.1326 \\
.0015\end{array}$ & $\begin{array}{c}63.0 \\
21.6 \\
68.9 \\
4.14\end{array}$ & $\begin{array}{l}20.5 \\
11.8 \\
59.4 \\
.89\end{array}$ & $\begin{array}{r}0.93 \\
.81 \\
.98 \\
.54\end{array}$ & $\begin{array}{c}7.69 \\
6.84 \\
11.8 \\
.75\end{array}$ & $\begin{array}{l}12 \\
32 \\
17 \\
18\end{array}$ & $\begin{array}{l}250-4200 \\
250-4200 \\
290-4200 \\
250-4200\end{array}$ \\
\hline $\begin{array}{l}\mathrm{HCO}_{3} \\
\mathrm{SO}_{4} \\
\mathrm{C} 1 \\
\mathrm{DS} \\
\mathrm{HRD}\end{array}$ & $\begin{array}{l}174 \\
170 \\
174 \\
164 \\
174\end{array}$ & $\begin{array}{l}194 \\
-32.93 \\
-81.97 \\
-14.68 \\
95.33\end{array}$ & $\begin{array}{l}.0068 \\
.1200 \\
.2490 \\
.5780 \\
.1847\end{array}$ & $\begin{array}{l}199 \\
65.4 \\
126 \\
460 \\
249\end{array}$ & $\begin{array}{l}46.1 \\
60.4 \\
119 \\
270 \\
95.1\end{array}$ & $\begin{array}{c}-.03 \\
.91 \\
.99 \\
.99+ \\
.92\end{array}$ & $\begin{array}{l}46.1 \\
24.9 \\
18.1 \\
24.9 \\
37.5\end{array}$ & $\begin{array}{r}23 \\
38 \\
14 \\
5 \\
15\end{array}$ & $\begin{array}{l}250-4200 \\
280-4200 \\
330-4200 \\
250-4200 \\
250-4200\end{array}$ \\
\hline \multicolumn{10}{|c|}{08164500 NAVIDAD RIVER NEAR GANADO, TEX. } \\
\hline $\begin{array}{l}\mathrm{Ca} \\
\mathrm{Mg} \\
\mathrm{Na} \\
\mathrm{Na} \\
\mathrm{K}\end{array}$ & $\begin{array}{r}212 \\
212 \\
156 \\
61 \\
67\end{array}$ & $\begin{array}{r}1.74 \\
.40 \\
-3.41 \\
-71.17 \\
3.02\end{array}$ & $\begin{array}{r}0.0934 \\
.1310 \\
.0942 \\
.1944 \\
.0013\end{array}$ & $\begin{array}{l}43.6 \\
6.27 \\
29.6 \\
65.5 \\
3.51\end{array}$ & $\begin{array}{c}21.7 \\
3.70 \\
14.5 \\
18.3 \\
.95\end{array}$ & $\begin{array}{l}.87 \\
.71 \\
.91 \\
.82 \\
.26\end{array}$ & $\begin{array}{c}10.8 \\
2.60 \\
6.10 \\
10.4 \\
.92\end{array}$ & $\begin{array}{l}25 \\
41 \\
21 \\
16 \\
26\end{array}$ & $\begin{array}{r}100-1050 \\
100-1050 \\
100-600 \\
601-1050 \\
100-1050\end{array}$ \\
\hline $\begin{array}{l}\mathrm{HCO}_{3} \\
\mathrm{SO}_{4} \\
\mathrm{Cl} \\
\mathrm{C} 1 \\
\mathrm{DS} \\
\mathrm{HRD}\end{array}$ & $\begin{array}{r}218 \\
222 \\
160 \\
62 \\
216 \\
218\end{array}$ & $\begin{array}{r}3.64 \\
5.42 \\
-9.01 \\
-76.02 \\
-2.14 \\
5.48\end{array}$ & $\begin{array}{l}.3448 \\
.0209 \\
.1377 \\
.2374 \\
.5831 \\
.2880\end{array}$ & $\begin{array}{l}157 \\
14.8 \\
39.3 \\
90.5 \\
258 \\
134\end{array}$ & $\begin{array}{c}72.6 \\
5.74 \\
20.5 \\
21.0 \\
118 \\
61.5\end{array}$ & $\begin{array}{l}.96 \\
.73 \\
.94 \\
.88 \\
.99+ \\
.95\end{array}$ & $\begin{array}{c}20.5 \\
3.92 \\
7.08 \\
10.0 \\
10.2 \\
20.1\end{array}$ & $\begin{array}{r}13 \\
26 \\
18 \\
11 \\
4 \\
15\end{array}$ & $\begin{array}{r}100-1050 \\
100-1050 \\
100-600 \\
601-1050 \\
100-1050 \\
100-1050\end{array}$ \\
\hline
\end{tabular}


Table 3. Results of regression analyses, $c_{i}=a_{i}+b_{i} k_{s c}$, for selected chemical constituents for 12 Texas stations, 1962-66 water years--Continued

\begin{tabular}{|c|c|c|c|c|c|c|c|c|c|c|}
\hline $\begin{array}{c}\text { Variable } \\
\text { (i) }\end{array}$ & $\begin{array}{l}\text { No. of } \\
\text { paired } \\
\text { values }\end{array}$ & $\begin{array}{c}\text { Intercept } \\
\qquad\left(a_{i}\right)\end{array}$ & $\begin{array}{l}\text { Regression } \\
\text { coefficient } \\
\qquad\left(b_{i}\right)\end{array}$ & $\begin{array}{c}\text { Mean } \\
\mathrm{C}_{i}\end{array}$ & $\begin{array}{c}\text { Standard } \\
\text { deviation } \\
\mathrm{C}_{i}\end{array}$ & $\begin{array}{l}\text { Correlation } \\
\text { coefficient } \\
\text { (r) }\end{array}$ & $\begin{array}{l}\text { Std. error } \\
\text { estimate } \\
\left(\mathrm{SE}_{\mathrm{e}}\right)\end{array}$ & $\begin{array}{l}\mathrm{SE} \text { as } \\
\text { percent } \\
\text { of mean }\end{array}$ & Range & in $\mathrm{K}_{\mathrm{SC}}$ \\
\hline \multicolumn{11}{|c|}{08176500 GUADALUPE RIVER AT VICTORIA, TEX. } \\
\hline $\begin{array}{l}\mathrm{Ca} \\
\mathrm{Mg} \\
\mathrm{Na} \\
\mathrm{K}\end{array}$ & $\begin{array}{r}133 \\
132 \\
134 \\
33\end{array}$ & $\begin{array}{r}11.00 \\
-6.05 \\
-6.56 \\
5.62\end{array}$ & $\begin{array}{r}0.0883 \\
.0400 \\
.0716 \\
.0054\end{array}$ & $\begin{array}{l}54.6 \\
13.7 \\
28.7 \\
3.01\end{array}$ & $\begin{array}{r}10.8 \\
4.52 \\
8.98 \\
.86\end{array}$ & $\begin{array}{r}0.83 \\
.90 \\
.81 \\
.68\end{array}$ & $\begin{array}{r}6.04 \\
2.00 \\
5.31 \\
.63\end{array}$ & $\begin{array}{l}11 \\
15 \\
19 \\
21\end{array}$ & $\begin{array}{l}200 \\
200 \\
200 \\
200\end{array}$ & $\begin{array}{r}725 \\
-\quad 725 \\
-\quad 725 \\
-\quad 725\end{array}$ \\
\hline $\begin{array}{l}\mathrm{HCO}_{3} \\
\mathrm{SO}_{4} \\
\mathrm{Cl} \\
\mathrm{Cl} \\
\mathrm{DS} \\
\mathrm{HRD}\end{array}$ & $\begin{array}{r}134 \\
134 \\
90 \\
44 \\
133 \\
134\end{array}$ & $\begin{array}{r}16.85 \\
-.31 \\
-13.74 \\
-67.79 \\
-6.26 \\
2.69\end{array}$ & $\begin{array}{r}.3843 \\
.0581 \\
.1046 \\
.1993 \\
.5923 \\
.3842\end{array}$ & $\begin{array}{c}206 \\
28.4 \\
33.1 \\
49.8 \\
286 \\
192\end{array}$ & $\begin{array}{c}42.9 \\
6.98 \\
10.5 \\
9.55 \\
61.1 \\
41.0\end{array}$ & $\begin{array}{l}.91 \\
.83 \\
.87 \\
.69 \\
.99 \\
.95\end{array}$ & $\begin{array}{c}18.2 \\
3.90 \\
5.19 \\
6.95 \\
10.4 \\
12.9\end{array}$ & $\begin{array}{r}9 \\
14 \\
16 \\
14 \\
4 \\
7\end{array}$ & $\begin{array}{l}200 \\
200 \\
200 \\
551 \\
200 \\
200\end{array}$. & $\begin{array}{l}725 \\
-\quad 725 \\
-\quad 550 \\
-\quad 725 \\
-\quad 725 \\
-\quad 725\end{array}$ \\
\hline \multicolumn{11}{|c|}{08188500 SAN ANTONIO RIVER AT GOLIAD, TEX. } \\
\hline $\begin{array}{l}\mathrm{Ca} \\
\mathrm{Mg} \\
\mathrm{Na} \\
\mathrm{K}\end{array}$ & $\begin{array}{r}169 \\
169 \\
171 \\
42\end{array}$ & $\begin{array}{r}14.53 \\
-2.65 \\
-15.15 \\
6.37\end{array}$ & $\begin{array}{r}0.0780 \\
.0228 \\
.1060 \\
.0001\end{array}$ & $\begin{array}{l}77.2 \\
15.7 \\
69.4 \\
6.30\end{array}$ & $\begin{aligned} 20.6 \\
6.03 \\
28.4 \\
1.24\end{aligned}$ & $\begin{array}{r}.96 \\
.96 \\
.96 \\
-.16\end{array}$ & $\begin{array}{l}6.03 \\
1.75 \\
8.13 \\
1.26\end{array}$ & $\begin{array}{r}8 \\
11 \\
12 \\
20\end{array}$ & $\begin{array}{l}200 \\
200 \\
200 \\
200\end{array}$ & $\begin{array}{l}-1300 \\
-1300 \\
-1300 \\
-1300\end{array}$ \\
\hline $\begin{array}{l}\mathrm{HCO}_{3} \\
\mathrm{SO}_{4} \\
\mathrm{Cl} \\
\mathrm{DS} \\
\mathrm{HRD}\end{array}$ & $\begin{array}{l}186 \\
185 \\
186 \\
170 \\
186\end{array}$ & $\begin{array}{r}51.36 \\
-12.31 \\
-27.75 \\
-9.31 \\
16.47\end{array}$ & $\begin{array}{r}.2282 \\
.1152 \\
.1457 \\
.6109 \\
.2978\end{array}$ & $\begin{array}{l}229 \\
77.1 \\
85.4 \\
480 \\
248\end{array}$ & $\begin{array}{c}63.3 \\
31.3 \\
39.8 \\
159 \\
80.6\end{array}$ & $\begin{array}{r}.95 \\
.97 \\
.97 \\
.98 \\
.98\end{array}$ & $\begin{array}{c}19.6 \\
7.30 \\
10.3 \\
32.1 \\
17.8\end{array}$ & $\begin{array}{r}9 \\
9 \\
12 \\
7 \\
7\end{array}$ & $\begin{array}{l}200 \\
200 \\
200 \\
200 \\
200\end{array}$ & $\begin{array}{l}-1300 \\
-1300 \\
-1300 \\
-1300 \\
-1300\end{array}$ \\
\hline
\end{tabular}


Although $\mathrm{SiO}_{2}$ (silica) is not appreciably ionized and does not directly affect the specific conductance, it is possible to have a significantly correlated $\mathrm{C}_{\mathrm{SiO}_{2}}{ }^{-\mathrm{K}_{\mathrm{Sc}}}$ relationship; as shown on figure 4 a for Trinity River at Rosser, Texas. This relationship may exist where most of the dissolved constituents, including silica, are from one source, such as a reservoir or ground water discharge and concentrations tend to vary proportionately. Providing no changes which would significantly alter the $\mathrm{C}_{\mathrm{SiO}_{2}}-\mathrm{K}_{\mathrm{Sc}}$ relationship take place within a basin, a regression equation that accounts for a major portion of the variation in the dependent variable may be used as a predictive tool over the range in values experienced during the calibration period. The $\mathrm{C}_{\mathrm{SiO}_{2}}{ }^{-\mathrm{K}_{\mathrm{SC}}}$ relations for most of the stations were poor, as exemplified by the graph for Guadalupe River at Victoria (fig. 4b). However, the basic statistics and plots do provide information on observed range and variation in concentrations experienced during the calibration period.

$\mathrm{NO}_{3}$ (nitrate) data and limited $\mathrm{PO}_{4}$ (phosphate) data generally show little relation to specific conductance, and range in concentrations are generally small for both variables (table 2). The one exception was for the Trinity River near Rosser. The quality of water at this station is greatly affected by municipal-waste discharge of the Dallas-Fort Worth metropolitan area. The significantly correlated $\mathrm{C}_{\mathrm{NO}_{3}}-\mathrm{K}_{\mathrm{Sc}}$ and $\mathrm{C}_{\mathrm{PO}_{4}}-\mathrm{K}_{\mathrm{Sc}}$ relationships (figs. $5 \mathrm{a}$ and $5 \mathrm{~b}$ ) exist because: (1) Streamflow of the Trinity River Basin above the Rosser station is highly regulated by water-supply reservoirs; (2) a large portion of the dissolved constituents, including $\mathrm{NO}_{3}$ and $\mathrm{PO}_{4}$, in water at the station are from municipal wastes; and (3) ranges in constituent concentrations and specific conductance result from dilution of fairly constant wastes discharges by variable runoff. 
With the regression equation developed in this study, $\mathrm{NO}_{3}$ and $\mathrm{PO}_{4}$ concentrations can be calculated from specific conductance with a standard error of estimate expressed as a percentage of the mean of 33 and 34 percent, respectively, for the Trinity River at Rosser (table 3). However, because these nutrients are important factors in water use and pollution control, more accurate determinations of these constituents may be required.

Limited fluoride observations in the data set generally show only minor variations in concentrations and no relation to specific conductance (fig. 6a). As shown in the basic statistics (table 2), the maximum range in fluoride concentration of 0.2 to $2.3 \mathrm{mg} / 1$ occurred at Trinity River near Rosser, with the higher concentrations probably resulting from waste inflows.

No relation of $\mathrm{pH}$ to specific conductance is shown at any of the stations (fig. 6b), and none would be expected in any Texas streams. Adequate $\mathrm{pH}-\mathrm{K}_{\mathrm{SC}}$ relationships probably do exist in some U.S. streams receiving high alkaline or acidic industrial wastes or acid mine drainage.

Although $\mathrm{C}_{i}-\mathrm{K}_{\mathrm{SC}}$ relationships for $\mathrm{SiO}_{2}, \mathrm{NO}_{3}, \mathrm{PO}_{4}, \mathrm{~F}$, and $\mathrm{pH}$ probably are poor for most surface-waters, the range in concentrations of these variables is generally limited in natural waters and where these ranges in concentrations have been determined by historical composite analyses, a reduced program to periodic analyses should serve most information needs. If one of these variables is 1 ikely to occur in concentrations that affect water use or exceed health or water-quality standards, analyses of discrete samples probably would be required to provide data for surveillance, control, or abatement procedures. 
Examples of highly-correlated $\mathrm{C}_{\mathrm{DS}}-\mathrm{K}_{\mathrm{SC}}$ and $\mathrm{C}_{\mathrm{Cl}}-\mathrm{K}_{\mathrm{SC}}$ relationships for 1962-66 water-year data are shown on figures $7 \mathrm{a}, 7 \mathrm{~b}, 8 \mathrm{a}$, and $8 \mathrm{~b}$. The

Figures $7 \mathrm{a}, 7 \mathrm{~b}, 8 \mathrm{a}$, and $8 \mathrm{~b}$ (captions on next page) belong near here. correlation coefficients $(r)$, presented in table 3, provide an index which measures the closeness of fit of observed points to the estimated regression line. Snedecor (1946, p. 148-155) discusses methodology that can be used to judge whether or not a computed $r$ is significantly different from zero. The following table presents the value of $r$ which must be equalled or exceeded at the 1 percent level of significance before the computed $r$ can be considered to be significantly different from zero (Snedecor, 1946).

\begin{tabular}{l|c}
\hline $\begin{array}{l}\text { Degrees of } \\
\text { freedom } \\
\text { (number of paired } \\
\text { observations -2) }\end{array}$ & $\begin{array}{c}\text { Value which computed } r \\
\text { must exceed before null } \\
\text { hypothesis, } \rho=0 \text { is rejected }\end{array}$ \\
\hline 10 & 0.708 \\
30 & .449 \\
50 & .354 \\
100 & .254 \\
150 & .208 \\
200 & .181 \\
\hline
\end{tabular}

The square of the correlation coefficient (coefficient of determination) is useful in explaining what percentage of the total variation is due to or accounted for by the regression. 


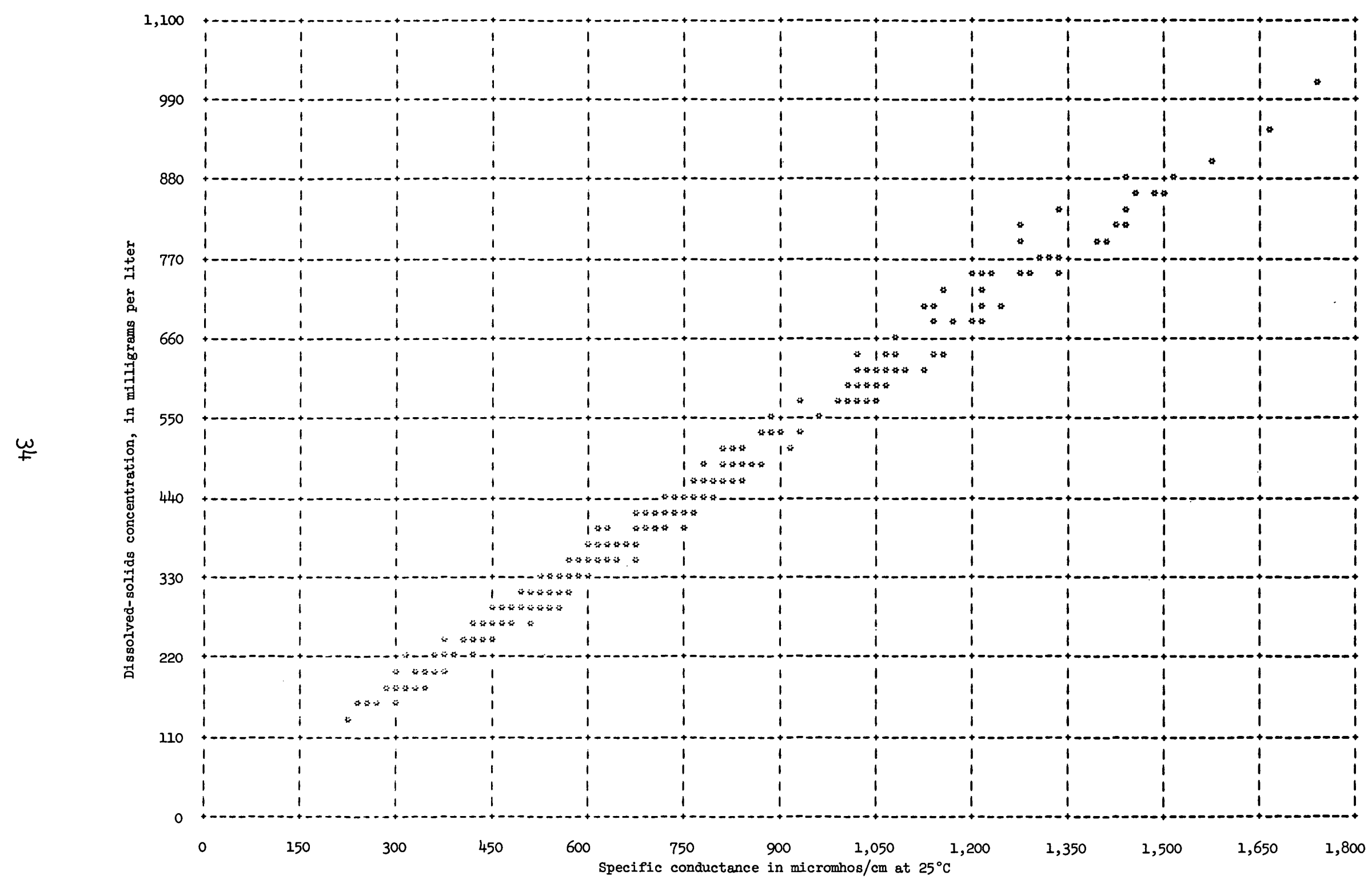

Figure 7a.--Dissolved solids versus specific conductance for station 08114000, Brazos River at Richmond, Tex. 


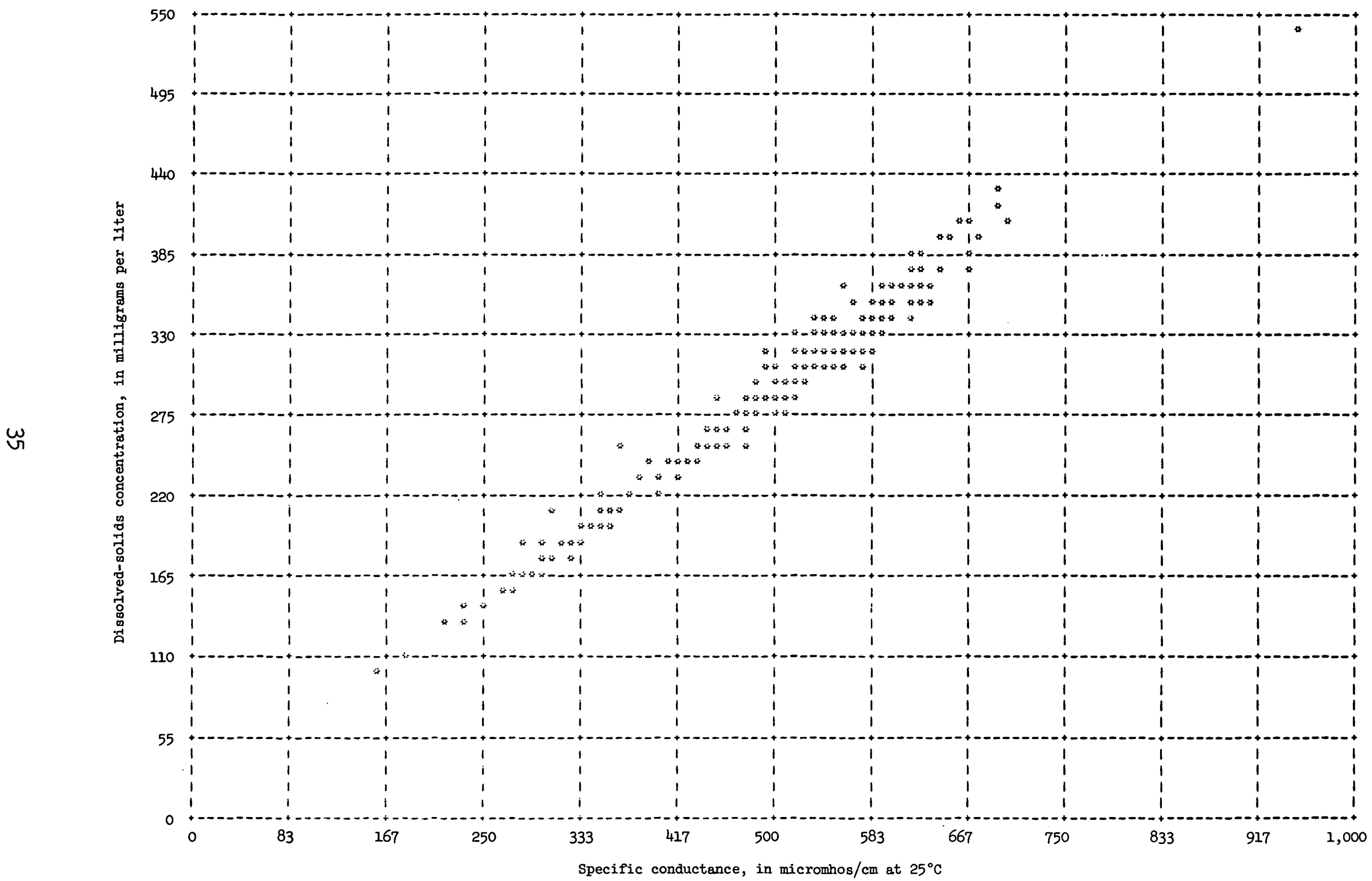

Figure Tb.--Dissolved solids versus specific conductance for station 08176500 , Guadalupe River at Victoria, Tex. 


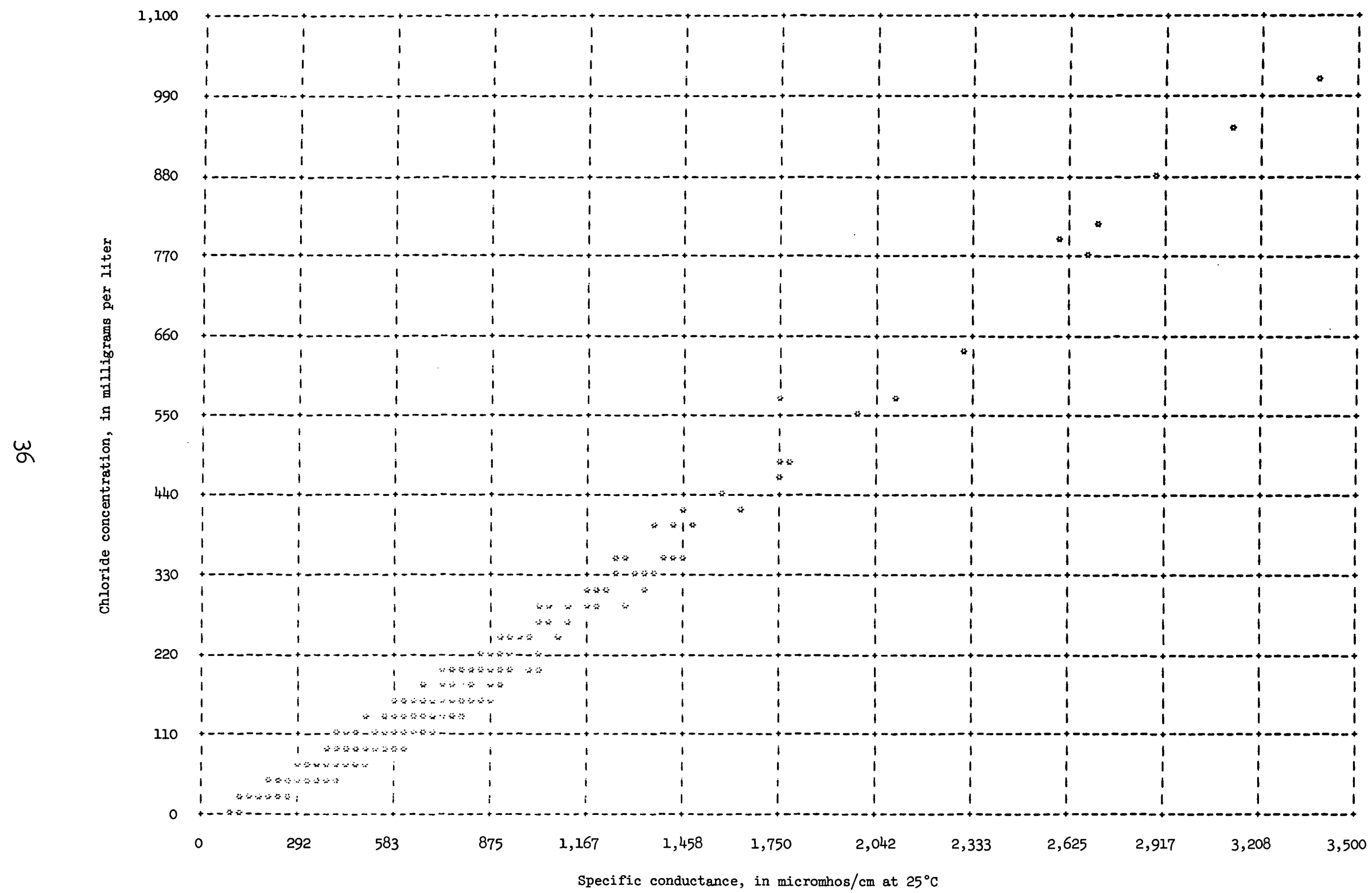

Figure 8a.--Chloride versus specific conductance for station 08111000, Navasota River near Bryan, Tex. 


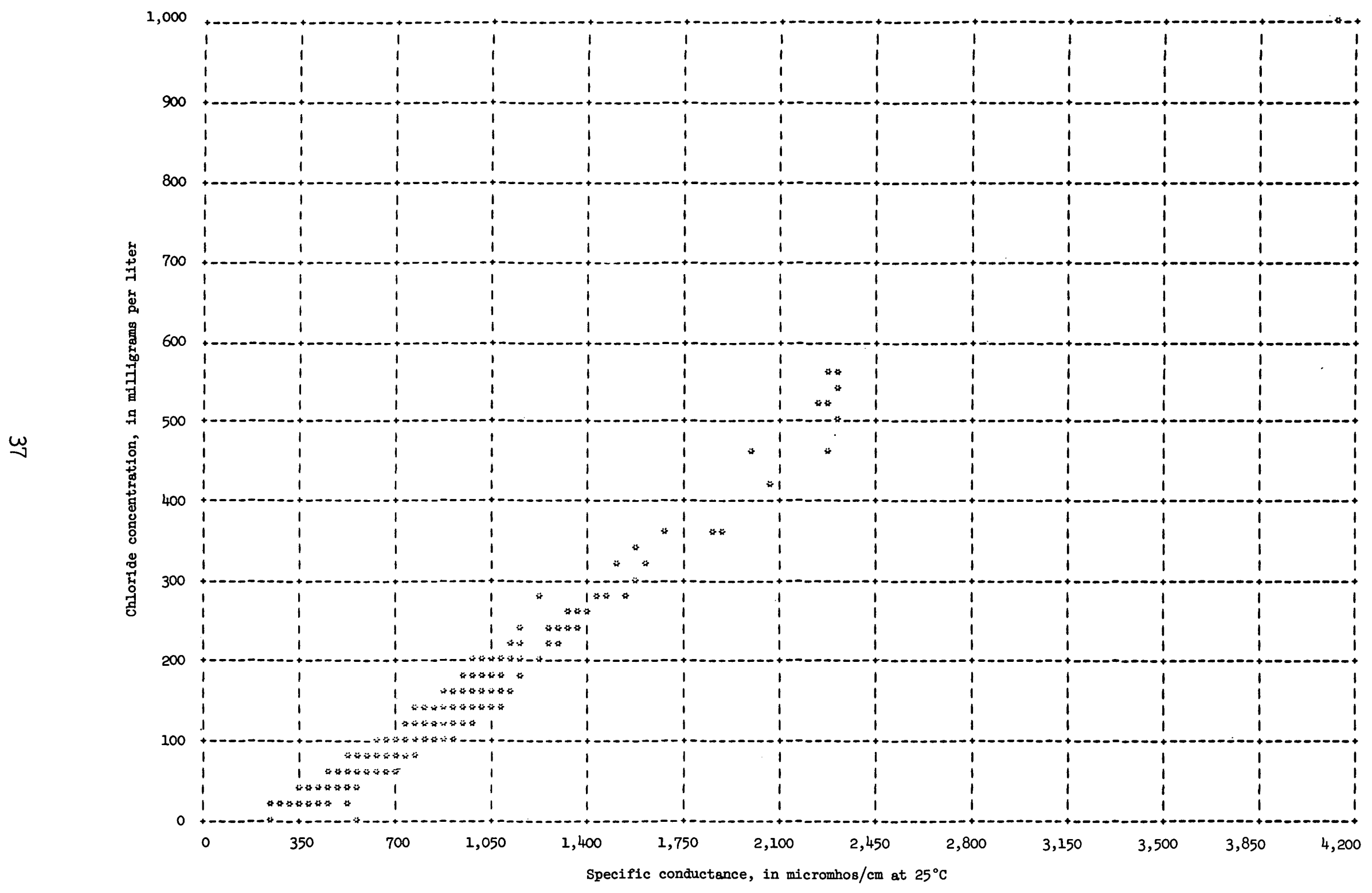

Figure 8b.--Chloride versus specific conductance for station 08147000, Colorado River near San Saba, Tex. 
Regression equations developed from the 1962-66 data sets for Ca (calcium), Mg (magnesium), $\mathrm{Na}$ (sodium), $\mathrm{K}$ (potassium), $\mathrm{HCO}_{3}$ (bicarbonate), $\mathrm{SO}_{4}$ (sulfate), $\mathrm{Cl}$ (chloride), and dissolved solids (table 3) were used in conjunction with daily water-discharge and specific-conductance values to simulate chemical-quality data for 169 station years (table 1). The simulated records include time- and discharge-weighted weekly, monthly, and annual summaries of concentrations and loads.

Annual discharge-weighted average concentrations were available for all station years in the historical data set, and these values were compared to the corresponding simulated values. All records outside the 1962-66 calibration period serve as test data for the regression. As an example, residuals obtained by substracting simulated from actual dischargeweighted concentrations (mg/l) for the Brazos River at Richmond are plotted on figure 9. Actual discharge-weighted concentrations of dissolved Figure 9 (caption on next page) belongs near here. solids versus time are also plotted on figure 9 to show extremes and yearly variations in total dissolved constituents. Actual and simulated annual discharge-weighted concentrations for the 1946-69 water-year period for this station are shown in table 4 . 

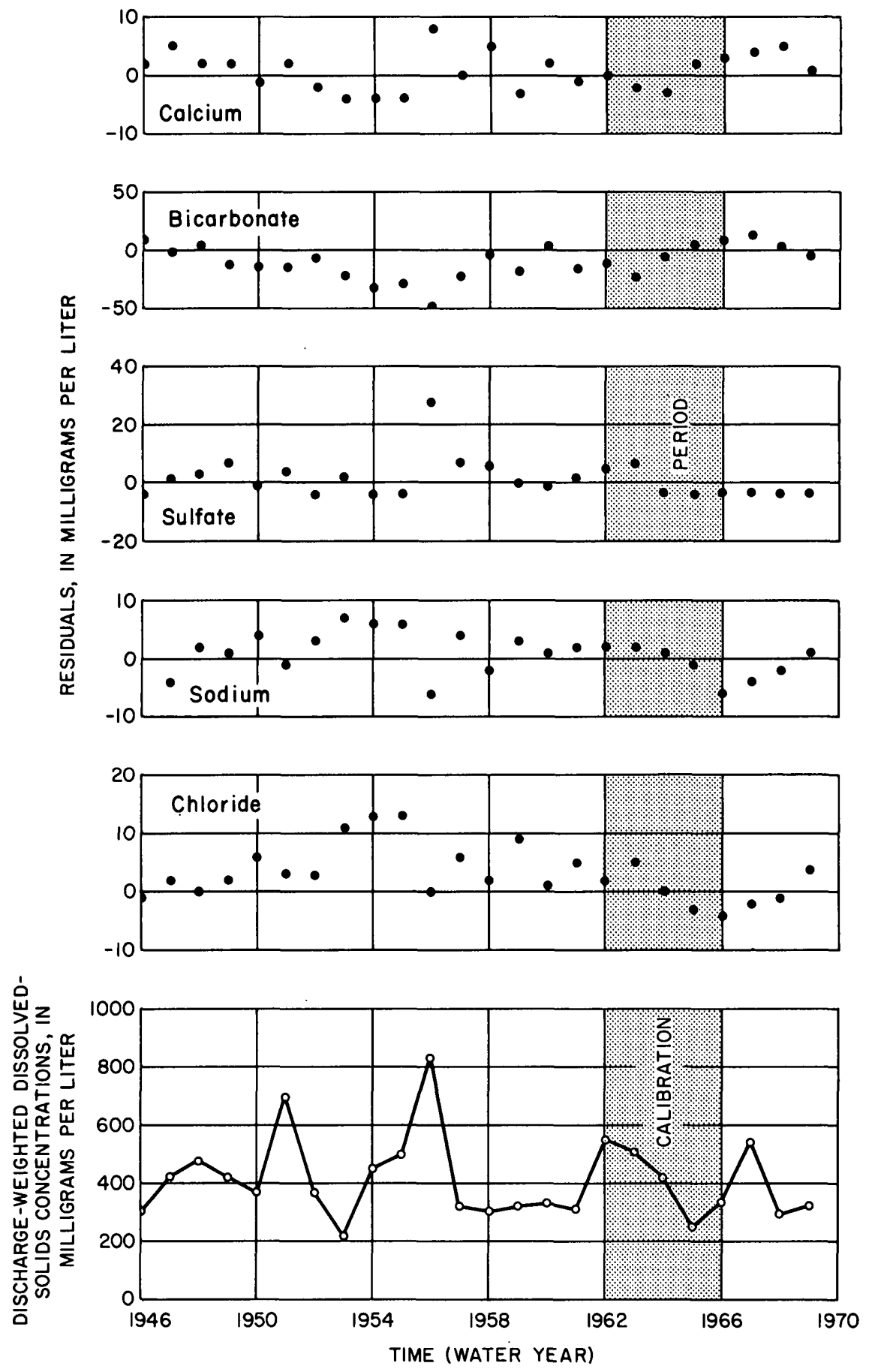

FIGURE 9.-Residuals in milligrams per liter (actual-simulated) for selected chemical constituents for the Brazos River at Richmond, Texas 
TABLE 4.--Actual and simulated annual discharge-weighted concentrations for the Brazos River at Richmond, Texas, 1946-69 water years

(Results in milligrams per 11ter)

\begin{tabular}{|c|c|c|c|c|c|c|c|c|c|c|c|c|c|c|c|c|c|c|c|c|c|c|c|c|c|}
\hline $\begin{array}{c}\text { Const1- } \\
\text { tuents }\end{array}$ & $\begin{array}{l}\text { Date } \\
\text { source }\end{array}$ & 1946 & 1947 & 1948 & 1949 & 1950 & 1951 & 1952 & 1953 & 1954 & 1955 & 1956 & 1957 & 1958 & 1959 & 1960 & 1961 & 1962 & 1963 & 1964 & 1965 & 1966 & 1967 & 1968 & 1969 \\
\hline \multirow[t]{2}{*}{$\mathrm{Ca}$} & Actual & 51 & 63 & 65 & 59 & 53 & 80 & 51 & 36 & 55 & 60 & 95 & 50 & 54 & 49 & 54 & 49 & 71 & 66 & 58 & 49 & 58 & 77 & 56 & 54 \\
\hline & Simulated & 49 & 58 & 63 & 57 & 54 & 78 & 53 & 40 & 59 & 64 & 87 & 50 & 49 & 52 & 52 & 50 & 71 & 68 & 61 & 47 & 55 & 73 & 51 & 53 \\
\hline \multirow[t]{2}{*}{$M g$} & Actual & 8.6 & 11 & 11 & 10 & 8.1 & 16 & 8.8 & 5.7 & 9.1 & 8.9 & 14 & 6.9 & 7.7 & 8.0 & 9.0 & 8.0 & 12 & 12 & 11 & 6.9 & 8.4 & 13 & 6.9 & 7.9 \\
\hline & Simulated & 7.6 & 10 & 11 & 10 & 9.1 & 16 & 9.1 & 5.5 & 11 & 12 & 19 & 7.9 & 7.6 & 8.3 & 8.3 & 7.9 & 13 & 13 & 11 & 7.1 & 9.0 & 14 & 8.0 & 8.6 \\
\hline \multirow[t]{2}{*}{$\mathrm{Na}$} & Actual & 37 & 63 & 82 & 70 & 60 & 139 & 60 & 23 & 83 & 95 & 166 & 46 & 37 & 49 & 48 & 44 & 106 & 97 & 77 & 32 & 48 & 101 & 41 & 51 \\
\hline & Simulated & 39 & 67 & 80 & 69 & 56 & 140 & 57 & 16 & 77 & 89 & 172 & 42 & 39 & 46 & 47 & 42 & 104 & 95 & 76 & 33 & 54 & 105 & 43 & 50 \\
\hline \multirow[t]{2}{*}{$\mathrm{k}$} & Actual 1 & -. & -- & -. & -- & -- & 2.4 & 2.8 & 3.8 & 4.1 & 4.9 & 5.8 & -. & 3.8 & 4.5 & -- & -- & -- & -. & -- & -- & -- & 4.4 & -- & -. \\
\hline & Simulated & 3.7 & 3.9 & 4.0 & 3.9 & 3.8 & 4.4 & 3.8 & 3.5 & 4.0 & 4.1 & 4.7 & 3.7 & 3.7 & 3.8 & 3.8 & 3.7 & 4.2 & 4.1 & 4.0 & 3.6 & 3.8 & 4.2 & 3.7 & 3.8 \\
\hline \multirow[t]{2}{*}{$\mathrm{HCO}_{3}$} & Actual & 155 & 152 & 162 & 141 & 136 & 160 & 143 & 115 & 124 & 132 & 136 & 124 & 142 & 130 & 151 & 132 & 153 & 140 & 151 & 149 & 159 & 180 & 150 & 145 \\
\hline & Simulated & 145 & 153 & 157 & 153 & 150 & 174 & 150 & 137 & 156 & 160 & 184 & 146 & 145 & 147 & 147 & 146 & 164 & 162 & 156 & 143 & 149 & 165 & 146 & 148 \\
\hline \multirow[t]{2}{*}{$\mathrm{SO}_{4}$} & Actual 1 & 39 & 70 & 84 & 76 & 58 & 134 & 54 & 25 & 72 & 83 & 185 & 54 & 50 & 51 & so & 49 & 106 & 100 & 74 & 35 & 54 & 100 & 45 & 51 \\
\hline & Simulated & 43 & 69 & 81 & 69 & 59 & 130 & 58 & 23 & 76 & 87 & 157 & 47 & 44 & 51 & 51 & 47 & 101 & 93 & 77 & 39 & 57 & 103 & 48 & 54 \\
\hline \multirow[t]{2}{*}{$\mathrm{cl}$} & Actual 1 & 53 & 100 & 118 & 103 & 87 & 214 & 85 & 31 & 127 & 145 & 260 & 65 & 57 & 74 & 67 & 64 & 156 & 145 & 111 & 43 & 72 & 153 & 60 & 75 \\
\hline & Simulated & 54 & 98 & 118 & 101 & 81 & 211 & 82 & 20 & 114 & 132 & 260 & 59 & 55 & 65 & 66 & 59 & 154 & 140 & 111 & 46 & 76 & 155 & 61 & 71 \\
\hline \multirow[t]{2}{*}{ DS } & Actual & 299 & 425 & 479 & 423 & 368 & 696 & 370 & 215 & 453 & 498 & 834 & 317 & 303 & 323 & 331 & 312 & 551 & 513 & 419 & 251 & 333 & 545 & 294 & 323 \\
\hline & Simulated & 275 & 392 & 448 & 395 & 346 & 678 & 344 & 180 & 426 & 479 & 804 & 290 & 279 & 308 & 311 & 290 & 543 & 506 & 429 & 254 & 340 & 553 & 296 & 323 \\
\hline
\end{tabular}


Residuals of calcium concentrations for the Brazos River at Richmond appean to be randomly scattered around the line of zero residual, indicating that the $\mathrm{C}_{\mathrm{Ca}}-\mathrm{K}_{\mathrm{SC}}$ relationship for the $1962-66$ period is valid over the 24 years of historical record. The difference in simulated calcium values was less than \pm 10 percent of the corresponding actual values for all years.

Bicarbonate residuals for this station were frequently negative, with simulated annual concentrations exceeding the actual in 14 of the 19 years in the test period (1946-61, 1967-69). Residuals (in percentage of actual) were usually less than 20 percent, except for 1956 when the $\mathrm{HCO}_{3}$ residual was -35 percent (actual $136 \mathrm{mg} / \mathrm{l}$, simulated $184 \mathrm{mg} / \mathrm{l}$ ). The discharge-weighted dissolved-solids concentration for 1956 was 834 $\mathrm{mg} / \mathrm{l}$, maximum for the period of record. The $\mathrm{C}_{\mathrm{HCO}_{3}}-\mathrm{K}_{\mathrm{Sc}}$ relationships were poor for 7 of the 12 stations, because maximum bicarbonate concentrations are more limited by saturation than other ions are, resulting in low $\mathrm{C}_{\mathrm{HCO}_{3}}{ }^{-\mathrm{K}_{\mathrm{Sc}}}$ correlations (see Steele, 1971).

Specific conductance may increase above a certain level with no increase, and in some waters a decrease, in $\mathrm{HCO}_{3}$ concentration. Regression equations for $\mathrm{C}_{\mathrm{HCO}_{3}}-\mathrm{K}_{\mathrm{Sc}}$ can probably be developed for many surface waters by segmenting the nonlinear rating curves into the approximate linear portions and developing regression equations for ranges in $\mathrm{K}_{\mathrm{SC}}$ within each of the line segments. For some surface waters, the maximum $\mathrm{HCO}_{3}$ concentration may be arbitrarily set as a constant when specific conductance values exceed the observed linear range for $\mathrm{C}_{\mathrm{HCO}_{3}}-\mathrm{K}_{\mathrm{SC}}$. 
Sulfate residuals were small and randomly scattered about the zero residual line. Difference in actual versus simulated val ues for sulfate was usually less than \pm 10 percent for the Brazos River station.

Although residuals for $\mathrm{Na}$ and $\mathrm{Cl}$ concentrations for Brazos River at Richmond were generally sma11, they were positive for most years before 1964. The change from positive to negative residuals in the mid-sixties may be due to a change in dissolved-constituent composition, but most likely results from random errors in simulation. The highest Na residual of $+7 \mathrm{mg} / 1$ (30 percent) and a high $\mathrm{Cl}$ residual of $+11 \mathrm{mg} / 1$ (35 percent) occurred in 1953 when the minimum annual weighted-average dissolved-solids concentration for the period of record occurred. $\mathrm{Na}$ and $\mathrm{Cl}$ residuals (in percentage of actual) were usually much less than \pm 10 percent for all other station years. Regression equations for $\mathrm{C}_{\mathrm{Na}}-$ and $\mathrm{C}_{\mathrm{Cl}^{-}}{ }^{-\mathrm{K}} \mathrm{Sc}$ developed for the upper and lower segments of the slightly nonlinear plots probably would improve the $\mathrm{C}_{i}-\mathrm{K}_{s c}$ relationships for $\mathrm{Na}$ and $\mathrm{Cl}$ concentrations for this station. 
Actual and simulated annual discharge-weighted concentrations for the Guadalupe River at Victoria, Texas, 1946-69 water years are shown in table 5. The plot of residuals for selected constituents for the Guadalupe River station (fig. 10) showed trends over time, indicating some

Figure 10 (caption on next page) belongs near here.

shifting in the $\mathrm{C}_{i}-\mathrm{K}_{\mathrm{SC}}$ regression relationships before the calibration period. Residuals (actual minus simulated) for $\mathrm{Ca}$ and $\mathrm{HCO}_{3}$ changed from negative to positive and $\mathrm{Na}$ and $\mathrm{Cl}$ residuals changed from positive to negative about the 1957 water year. Rawson (1968, p. 11) discussed the change in chemical composition, the decline in $\mathrm{Na}$ and $\mathrm{Cl}$ concentrations, and the resulting shift in $\mathrm{Ca}-\mathrm{HCO}_{3}$ and $\mathrm{Na}-\mathrm{Cl}$ relationships to specific conductance.

Since the mid-fifties, the volume of oil-field brines reaching the streams in the upper Juadalupe Basin has been reduced, with all or most of these brines now being injected in deep wells. As shown by the residual plots in figure 9, systematic errors are caused by using the regression equations developed from the 1962-66 calibration period for simulating records during the 1946-57 water year period for this station. If regression equations are to be used to compute chemical concentrations and loads for current data-collection activities, sufficient laboratory analysis of samples should be included in the continuing data-collection program to provide periodic checks of the $\mathrm{C}_{i}-\mathrm{K}_{\mathrm{SC}}$ relationships. Revised $\mathrm{C}_{i}-\mathrm{K}_{\mathrm{SC}}$ regression equations should be developed when any significant shifts are detected for any of the constituents. 

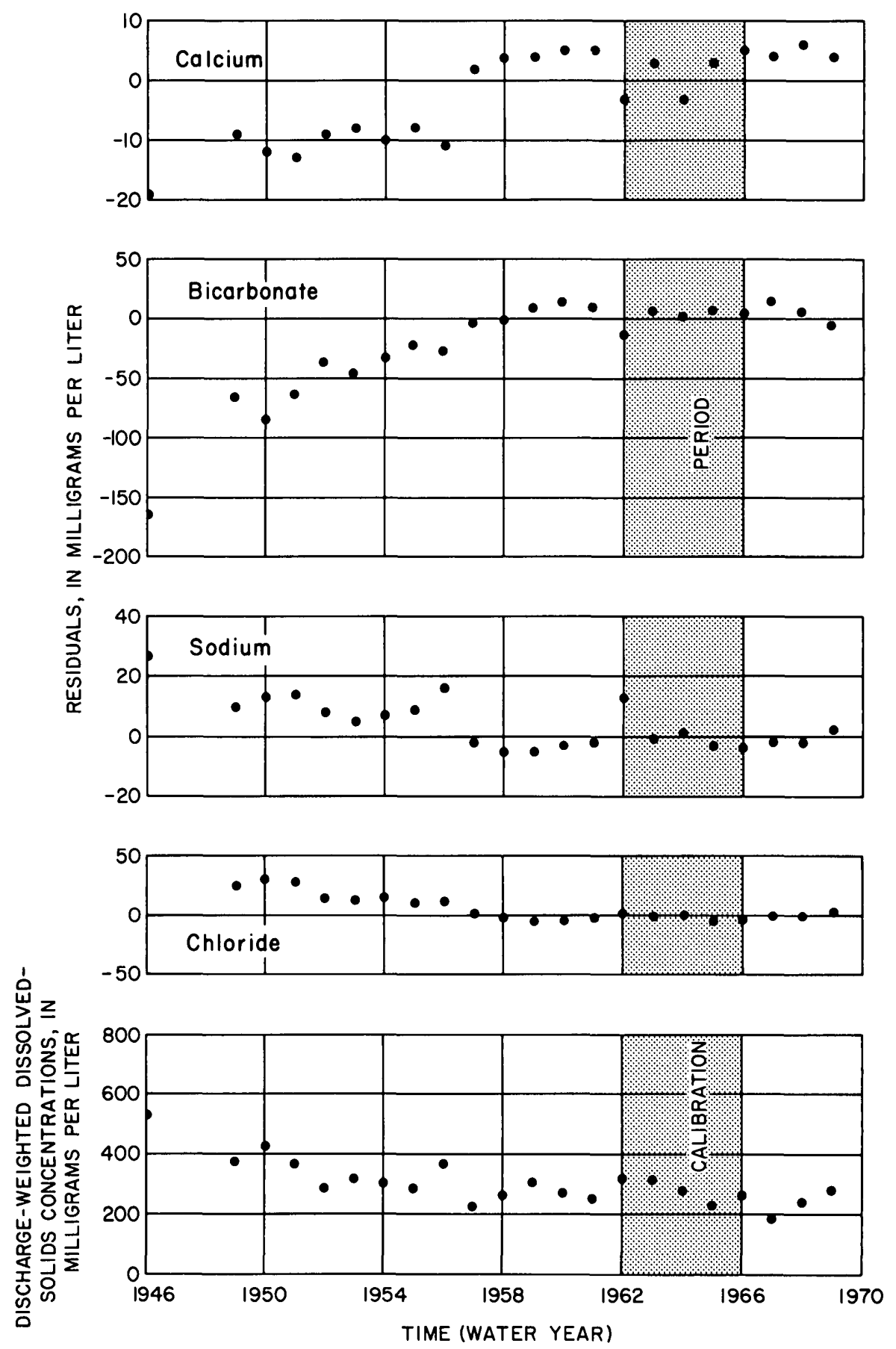

FIGURE 10.- Residuals in milligrams per liter (actual-simulated) for selected chemical constituents for the Guadalupe River at Victoria, Texas 
TABLE 5.--Actual and simulated annual discharge-weighted concentrations for the Guadalupe River at Victoria, Texas, 1946-69 water years

\begin{tabular}{|c|c|c|c|c|c|c|c|c|c|c|c|c|c|c|c|c|c|c|c|c|c|c|c|c|c|}
\hline $\begin{array}{c}\text { Const1- } \\
\text { tuents }\end{array}$ & $\begin{array}{c}\text { Date } \\
\text { Bource }\end{array}$ & 1946 & 1947 & 1948 & 1949 & 1950 & 1951 & 1952 & 1953 & 1954 & 1955 & 1956 & 1957 & 1958 & 1959 & 1960 & 1961 & 1962 & 1963 & 1964 & 1965 & 1966 & 1967 & 1968 & 1969 \\
\hline \multirow[t]{2}{*}{$\mathrm{S}_{10} \mathrm{O}_{2}$} & Actual & -- & -- & $-\cdot$ & 14 & 15 & 16 & 17 & .17 & 19 & 18 & 16 & 13 & 14 & 15 & 16 & 15 & 17 & 15 & 13 & 11 & 11 & 9.5 & 11 & 12 \\
\hline & Simulated & 18 & 18 & 22 & 15 & 16 & 15 & 14 & 14 & 14 & 14 & 15 & 12 & 13 & 14 & 14 & 13 & 14 & 14 & 14 & 13 & 14 & 12 & 13 & 14 \\
\hline \multirow[t]{2}{*}{$\mathrm{Ca}$} & Actua 1 & 69 & -. & -- & 57 & 60 & 53 & 45 & 51 & 46 & 46 & 56 & 45 & 53 & 60 & 58 & 53 & 55 & 61 & 51 & 51 & 57 & 44 & 54 & 57 \\
\hline & Simulated & 88 & 85 & 118 & 66 & 72 & 66 & 54 & 59 & 56 & 54 & 67 & 43 & 49 & 56 & 53 & 48 & 58 & 58 & 54 & 48 & 52 & 40 & 48 & 53 \\
\hline \multirow[t]{2}{*}{ Mg } & Actua 1 & 18 & $-\cdot$ & .. & 15 & 17 & 17 & 12 & 14 & 14 & 12 & 16 & 7.3 & 11 & 14 & 13 & 11 & 16 & 15 & 14 & 10 & 12 & 7.6 & 9.8 & 11 \\
\hline & Simulated & 29 & 27 & 43 & 19 & 22 & 19 & 13 & 15 & 14 & 14 & 20 & 8.6 & 11 & 14 & 13 & 11 & 15 & 15 & 13 & 10 & 13 & 7.2 & 11 & 13 \\
\hline \multirow[t]{2}{*}{$\mathrm{Na}$} & Actual & 83 & -- & -. & 48 & 56 & 52 & 36 & 37 & 37 & 38 & 55 & 18 & 20 & 25 & 25 & 22 & 35 & 31 & 29 & 20 & 23 & 15 & 22 & 29 \\
\hline & Simulated & 56 & 53 & 80 & 38 & 43 & 38 & 28 & 32 & 30 & 29 & 39 & 20 & 25 & 30 & 28 & 24 & 32 & 32 & 28 & 23 & 27 & 17 & 24 & 27 \\
\hline \multirow[t]{2}{*}{$\mathrm{k}$} & Actual & -- & -- & -- & -- & -. & 1.0 & 2.8 & 3.7 & 3.4 & 3.6 & 3.9 & 4.5 & 3.3 & 2.8 & -- & -- & -- & -. & -- & -- & 3.0 & 3.2 & -. & -- \\
\hline & Simulated & 10 & 10 & 12 & 9.0 & 9.4 & 9.0 & 8.2 & 8.5 & 8.4 & 8.3 & 9.1 & 7.6 & 8.0 & 8.4 & 8.2 & 7.9 & 8.5 & 8.5 & 8.2 & 7.9 & 8.2 & 7.4 & 7.9 & 8.2 \\
\hline \multirow[t]{2}{*}{$\mathrm{HCO}_{3}$} & Actual 1 & 191 & -- & -- & 190 & 199 & 195 & 166 & 179 & 179 & 184 & 235 & 153 & 183 & 219 & 215 & 188 & 210 & 230 & 203 & 183 & 202 & 158 & 185 & 193 \\
\hline & Simulated & 354 & 338 & 484 & 255 & 283 & 258 & 202 & 224 & 212 & 206 & 262 & 157 & 184 & 211 & 201 & 178 & 223 & 223 & 202 & 176 & 197 & 144 & 179 & 199 \\
\hline \multirow[t]{2}{*}{$\mathrm{SO}_{4}$} & Actual & 39 & -- & -- & 28 & 32 & 30 & 24 & 29 & 27 & 27 & 30 & 21 & 27 & 28 & 27 & 24 & 34 & 31 & 26 & 24 & 27 & 15 & 25 & 31 \\
\hline & Simulated & 51 & 48 & 70 & 36 & 40 & 36 & 28 & 31 & 29 & 28 & 37 & 21 & 25 & 29 & 28 & 24 & 31 & 31 & 28 & 24 & 27 & 19 & 24 & 27 \\
\hline \multirow[t]{2}{*}{$\mathrm{Cl}$} & Actual & 160 & $-\cdot$ & -- & 86 & 104 & 89 & 56 & 61 & 58 & 51 & 72 & 26 & 31 & $35^{\circ}$ & 33 & 29 & 47 & 42 & 37 & 26 & 33 & 20 & 30 & 40 \\
\hline & Simulated & 109 & 101 & 176 & 61 & 73 & 61 & 42 & 48 & 43 & 40 & 60 & 25 & 33 & 40 & 37 & 32 & 45 & 43 & 37 & 30 & 36 & 21 & 31 & 38 \\
\hline \multirow[t]{2}{*}{ DS } & Actual 1 & 532 & -- & -. & 380 & 425 & 371 & 291 & 319 & 304 & 293 & 368 & 227 & 264 & 303 & 288 & 258 & 321 & 316 & 281 & 236 & 266 & 191 & 246 & 284 \\
\hline & S1mulated & 513 & 488 & 714 & 361 & 405 & 366 & 279 & 312 & 295 & 285 & 372 & 210 & 252 & 293 & 278 & 243 & 312 & 311 & 279 & 239 & 272 & 190 & 244 & 275 \\
\hline
\end{tabular}


The comparison of simulated data to historical records, computed from composite samples, tests the predictive capability of the $\mathrm{C}_{\mathrm{i}}-\mathrm{K}_{\mathrm{sc}}$ regression equations and indicates the levels of accuracy that can be expected if regression equations and daily specific-conductance values are used conjunctively to produce current information on chemical quality. This comparison also points out changes in the relative abundances of ions if and when changes in composition occur. The differences between simulated and actual constituent values are influenced by random errors, and may be influenced by compositional changes. However, the comparisons also appear to be partly biased by differences between the composite and daily specific-conductance records.

Figure 11 shows a plot of annual mean discharge-weighted specific Figure 11 (caption on next page) belongs near here. conductance values from composites versus annual mean discharge-weighted specific conductance values from daily samples for the Neches River at Evadale. The relationship is very good, but most of the data points fall above the line of equivalence (intercept $=0$, slope $=1.00$ ), showing annual averages from composites to be consistently, but only slightly higher than those from daily values.

The higher discharge-weighted specific-conductance values for periods of similar specific-conductance results from use of equal volumes of the daily samples instead of volumes proportional to discharge. Compositing by equal volume results in a time-weighted sample for the composite period, which is likely to be slightly higher than a discharge-weighted composite for the same period. 


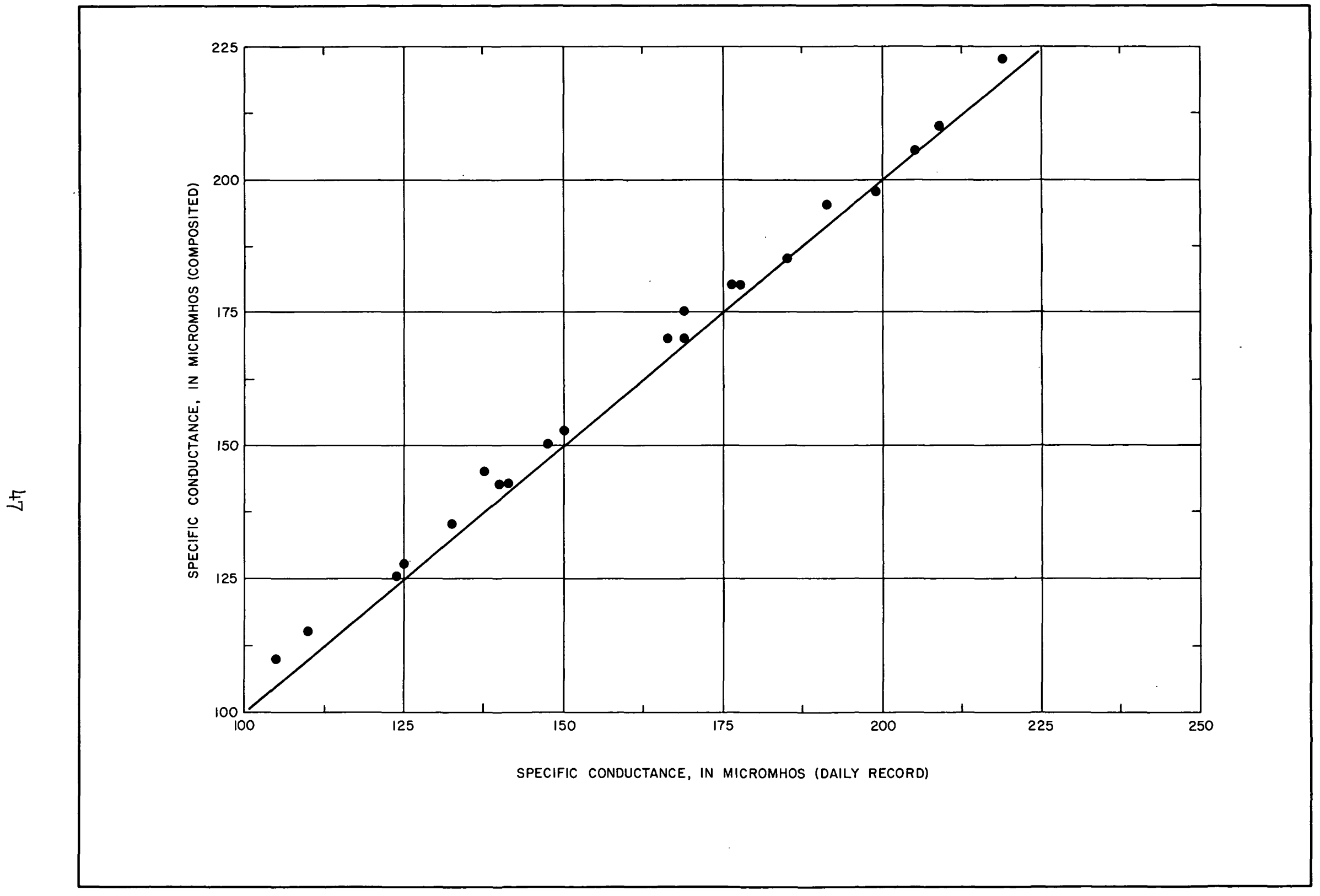

FIGURE II.-Annual discharge-weighted specific conductance values from composite samples versus values from daily samples, Neches River at Evadale 
Annual mean discharge-weighted specific conductance values from composited samples are actually discharge-weighted values for a group of time weighted composites. Of course, the same thing is done when one daily value is used to represent the discharge for that day--only the time-weighting period is shortened. The purpose of this discussion is not to criticize past or present data-collection activities, but to point out one source of bias not associated with the regression equations that may account for some of the differences between actual and simulated values reported in this study.

The only significant change in the relative abundance of ions observed in the analysis of streamflow chemical-quality data for the 12 stations was for the Guadalupe River at Victoria (fig. 10). Figure 12

Figure 12 (caption on next page) belongs near here.

shows actual and simulated annual mean discharge-weighted concentrations of calcium, chloride, and dissolved solids for the Neches River at Evadale, Texas. Actual and simulated values for calcium and chloride agree very well, and the plots show no time trends over the 22-year period. However, the actual dissolved-solids concentrations are greater than the simulated values from 1948 through 1955 and much greater during $1948-50$ 


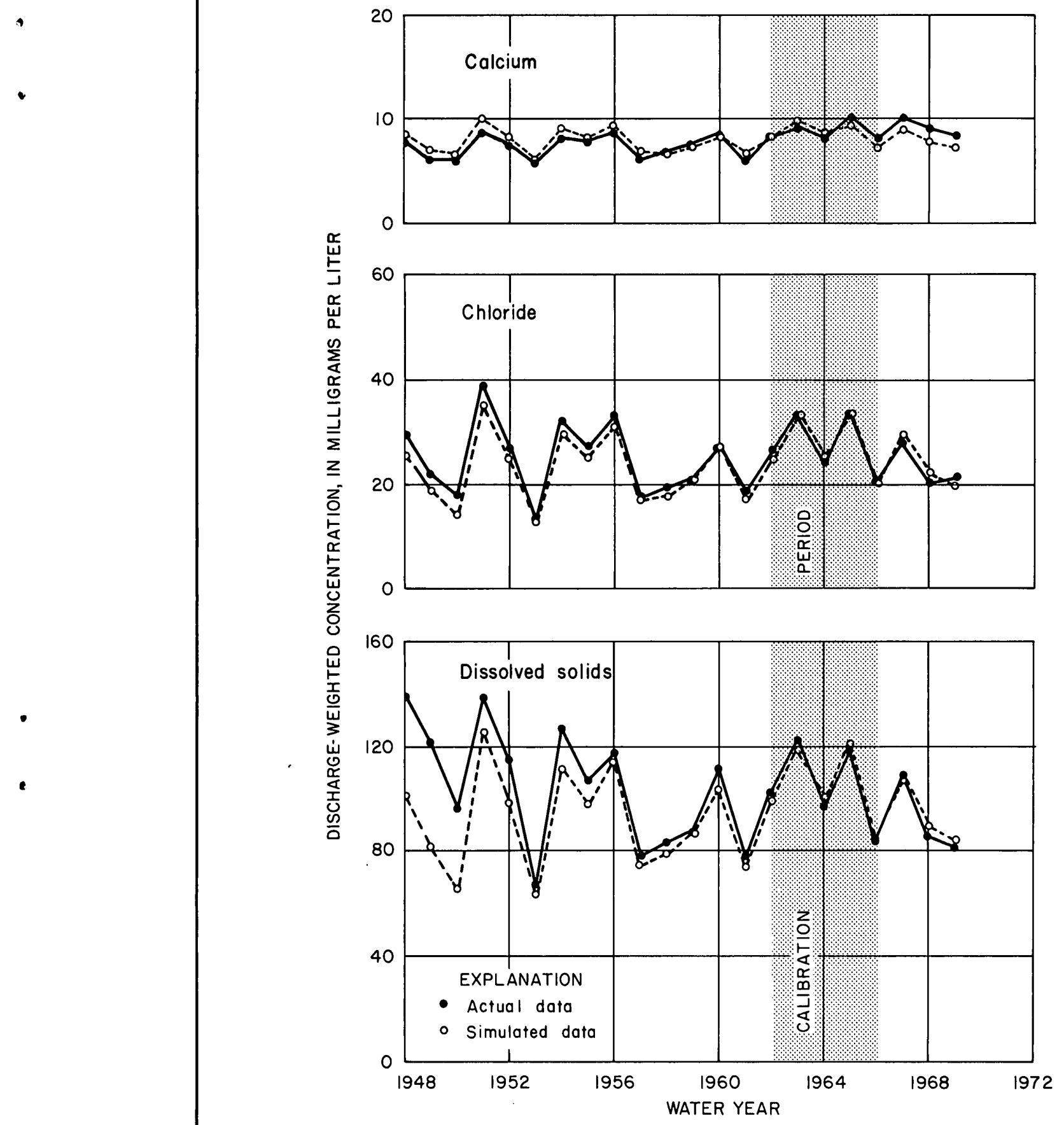

FIGURE 12. - Actual and simulated discharge-weighted concentrations of calcium, chloride, and dissolved solids for Neches River at Evadale, Texas 
The high positive residuals (actual minus simulated concentrations) indicated that the $\mathrm{C}_{\mathrm{DS}}-\mathrm{K}_{\mathrm{SC}}$ regression equation developed from the $1962-$ 66 calibration period simulates a record for the $1948-55$ test period with errors as large as 30 percent, suggesting a decrease in dissolvedsolids concentrations relative to the level of specific conductance over time. However, the consistently positive residuals during the 1948-55 test period result to a large extent from a change in analytical procedures for determining dissolved-solids concentrations.

The dissolved-solids concentrations used in developing the regression equations were all calculated sums of determined constituents; whereas, the majority of dissolved-solids concentrations reported from 1948-55 were determined by residue on evaporation. The residue values at this particular sampling location include dissolved organic materials and probably average 10-20 percent higher than the sum of determined constituents. The differences in annual specific conductance values (fig. 11) also would account for part of the discrepancy shown in figure 12. In the analysis of historical data and in the application of regression equations for simulating chemical quality, problems of this nature must be taken into account in testing the adequacy of the regression procedure. 


\section{SUMMARY AND CONCLUSIONS}

The water-quality records for the 12 stations evaluated in this study are considered to be representative of the variability in location and in the level of salinity of water of Texas streams. Concentration-discharge plots generally show poor relationships for all of the selected stations and should be an indication of the limitations for prediction of solute concentrations from water discharge in Texas streams. On the other hand, concentration-specific conductance plots and subsequent regression equations developed for each station using data from the 1962-66 water years and testing with available data outside this period indicate that specific conductance can be used as an index variable for estimating selected solute concentrations in Texas streams and that this procedure, with few exceptions, can be used as an alternative to composite analyses for calculation of solute concentrations and loads.

Results of regression analyses and comparisons of simulated versus actual data indicate that specific conductance records can be used in conjunction with $\mathrm{C}_{i}-\mathrm{K}_{\mathrm{Sc}}$ regression equations (table 3 ) to calculate annual dissolved-solids concentrations and loads within 10 percent of results from laboratory values and annual $\mathrm{Ca}, \mathrm{Mg}, \mathrm{Na}, \mathrm{Cl}$, and $\mathrm{SO}_{4}$ concentrations and loads usually within less than 20 percent. By developing regression equations for segments of the $C_{i}-K_{s c}$ relationships for each station, accuracy of estimation can be improved for several of the cases studies. Estimates of $\mathrm{HCO}_{3}$ concentrations can be improved by using two or more regression equations over the observed $\mathrm{K}_{\mathrm{sc}}$ range or by using the maximum $\mathrm{HCO}_{3}$ value as a constant for all $\mathrm{K}_{\mathrm{SC}}$ values above a determined level as indicated by the historical data. 
As might be expected, the $\mathrm{C}_{i}-\mathrm{K}_{\mathrm{SC}}$ relationships were generally poor for $\mathrm{SiO}_{2}, \mathrm{~F}, \mathrm{NO}_{3}, \mathrm{PO}_{4}$, and $\mathrm{pH}$; but the observed range in concentrations of these constituents were usually very limited, and variability in concentration has been fairly well defined by the historical data. If these constituents are likely to be present in concentrations that exceed water-quality standards or limit water use, analysis of individual samples at a determined sampling frequency should be continued in the data-collection program to monitor the problem or potential problem. Otherwise, data collection for these constituents should be reduced. The use of specific conductance as an index variable in conjunction with concentration-specific conductance regression equations is a flexible system that can be applied with sufficient accuracy to satisfy most anticipated needs for data. For water management, daily specificconductance determinations, or continuous specific-conductance records from monitors, accompanied by periodic analyses of the inorganic constituents to serve as continued checks on the regressions, would probably be required. For stations providing data for planning and design purposes, less frequent measurements of specific conductance may be sufficient along with the periodic check chemical analyses. 
This report summarizes and discusses the results of several dataanalysis procedures for historical water-quality records for 12 Texas stations. As of the 1971 water year, over 60 additional Texas stations, each having between six and 33 years of record, have data amenable for the type of analysis used in this report. If these records were analyzed using the procedures prescribed in this study, additional techniques, such as regionalization of chemical-quality conditions and an assessment of frequency-of-sampling alternatives, could be tested.

A change in station activities from composite chemical analyses to estimated values of solute concentrations and loads using simulative techniques should result in decreased costs and manpower requirements for this one facet of the water-quality data-collection programs. The resources made available by this reorientation may well be applied to other areas of water-quality programs currently constrained by lack of adḑitional manpower and laboratory facilities. Net savings will depend both on information needs and specified accuracy criteria that are required in the overall water-quality program. 
Colby, B. R., Hembree, C. H., and Rainwater, F. H., 1956, Sedimentation and chemical quality of surface waters in the Wind River basin, Wyoming: U.S. Geol. Survey Water Supply Paper 1373, 336 p.

Leifeste, D. K., Blakey, J. F., Hughes, L. S., 1971, Reconnaissance of the chemical quality of surface waters of the Red River basin, Texas: Texas Water Deve1. Board Rept. 129, 72 p.

Rawson, Jack, 1967, Study and interpretation of chemical quality of surface waters in the Brazos River basin, Texas: Texas Water Devel. Board Rept. 55, 113 p.

1968, Reconnaissance of the chemical quality of surface waters of the Guadalupe River basin, Texas: Texas Water Devel. Board Rept. 88,37 p.

Snedecor, G. W., 1946, Statistical methods: Iowa, Iowa State College Press, p. 148-155.

Steele, T. D., 1970, Beneficial uses and pitfalls of historical waterquality data: Proceeding of national symposium on data and instrumentation for water-quality management, Conference of State Sanitary Engrs., Madison, Wis., July 21-23, 1970, p. 346-363

1971, A study of the chemical quality of streamflow in Arkansas: U.S. Geol: Survey open file report, 40 p. 